\title{
Simulated Annealing Clusterization Algorithm for Studying the Multifragmentation.
}

\author{
Rajeev K. Puri周 and Joerg Aichelin \\ SUBATECH, Laboratoire de Physique Subatomique et des Technologies Associees \\ UMR Universite de Nantes, IN2P3/CNRS, Ecole des Mines de Nantes \\ 4 rue Alfred Kastler, F-44070 Nantes, France.
}

\begin{abstract}
We here present the details of the numerical realization of the recently advanced algorithm developed to identify the fragmentation in heavy ion reactions. This new algorithm is based on the Simulated Annealing method and is dubbed as Simulated Annealing Clusterization Algorithm [SACA]. We discuss the different parameters used in the Simulated Annealing method and present an economical set of the parameters which is based on the extensive analysis carried out for the central and peripheral collisions of $\mathrm{Au}-\mathrm{Au}, \mathrm{Nb}-\mathrm{Nb}$ and $\mathrm{Pb}-\mathrm{Pb}$. These parameters are crucial for the success of the algorithm. Our set of optimized parameters gives the same results as the most conservative choice, but is very fast. We also discuss the nucleon and fragment exchange processes which are very important for the energy minimization and finally present the analysis of the reaction dynamics using the new algorithm. This algorithm is can be applied whenever one wants to identify which of a given number of constituents form bound objects.
\end{abstract}

*Permanent address: Physics Department, Panjab University, Chandigarh-160 014, India 


\section{Introduction:}

In recent years, a lot of efforts has been made (in experiments and theory) at low, intermediate and relativistic energies to understand the physics which drives heavy ion reactions. A new generation of electronic devices made it possible to measure a multitude of observables at the same time which can give information about the hot and dense nuclear matter formed during a reaction [1]. In a heavy ion reaction the density can be as high as 2-4 times the normal nuclear matter and one may reach temperatures of about $100 \mathrm{MeV}$ [2]. The properties of the nuclear matter at high densities are not only of importance for nuclear physics, but are also of great use for the astrophysical studies especially for supernova studies. Unfortunately, there is no method to measure directly the properties of hot and compressed nuclear matter formed during a reaction [1]. What one can observe are single hadrons. Their properties are mostly determined in the late stage of the expansion and it is quite difficult to find observables sensitive to the early stage. For this one has to rely on the theoretical (simulation) models. One can simulate the reaction from the start to the end where we find cold nuclear matter in the form of nucleons, light and heavy fragments [3]. The most important information which one would like to extract from the simulation are the time scales of different phenomena.

One would like to know, for example, when particles are created, when fragments are formed, whether they carry any information about the hot and compress phase etc.? The key question associated with the time scale of the fragment emission is whether it is a thermal or a dynamical process i.e. whether the fragments are created after the system has thermalized or can already be recognized early, before a possible thermalization sets in . This would point to initial-final state correlations $\llbracket 4, ?, ?, ?]$. In addition, several conjectures on the equation of state, especially those in which the nuclear interaction is strongly momentum dependent, could not be tested so far in simulations because the nuclei become unstable. Here an early fragment recognization would allow to study these equations of state.

We shall concentrate here on multifragmentation. All theoretical models used to study heavy ion collisions are based on the transport nucleons and mesons only. Therefore, for the study of multifragmentation a method has to be deviced to group the nucleons into free nucleons and fragments. In the past, one has taken the spatial correlations among nucleons to group them into fragments [3]. Naturally, this approach cannot detect different fragments which are (almost) overlapping and therefore will give a single big fragment during the early stage of the reaction where density is quite high. In other words, simple coordinate space approaches cannot address the question of the time 
scale of fragment formation. To study that one needs a method where fragments can be identified even if they are overlapping, i.e. methods which are based on phase space.

We conjecture that in nature at any given moment of the reaction that configuration is realized which gives the largest binding energy. That this concept is meaningful and gives sensitive results will be demonstrated later. To find the most bound configuration we are confronted with two problems.

a) The huge number of possible configurations.

b) The fact that the number of entities changes. Whereas the number of nucleons is constant, the number of free nucleons and fragments is a variable. The problem caused by this fact will be discussed later.

One may approach this problem by simple iterative methods. They, however, do not garranty that a global minimum is obtained but may arrive at a local minimum [8]. First attempt to overcome this problem has been advanced in ref. [6]. Though this method works fine for small systems, its simple numerical implementation poses serious problems for studying the heavy systems where the number of different configurations increases tremendously and almost always the algorithm remained stuck in a local minima. To deal with the more interesting large systems a sophisticated algorithm is needed which can handle the huge number of different configurations and finds the configuration with maximal binding energy in a reasonable amount of computational time. In addition, it should be able to overcome any type of local minima.

We here present the details and technical aspects of such a new algorithm which is based on the simulated annealing method and is quite general in nature. The question addressed here requires an numerical approach which is not specific to the problem described. Apart from multifragmentation the energy minimization is needed, for example, in the nuclear structure calculations, in cluster radioactivity, in hadron physics etc. In nuclear cluster radioactivity, one is interested in the relative yields of different fragments which are emitted by the decaying nucleus. There, one assumes the isotopic distribution and the energy minimization is needed to find the most bound isobaric distribution [9.

Naturally, before one can talk about cluster formation, one first needs the phase space coordinates of the particles. 
We here use the Quantum Molecular Dynamics (QMD) model [3] (as an event generator) to generate the time evolution of the phase space coordinates of nucleons in a nuclear reaction.

Our paper is organized as follow: The section II deals with the short description of the QMD model and a detailed description of the algorithm . The numerical realization of the algorithm is presented in section III and we summarize the results in section IV.

\section{The Formalism}

We here summarize shortly the Quantum Molecular Dynamics [QMD] model and then give the details of our new algorithm designed for multifragmentation. For more details on the QMD approach, we refer the reader to [3].

\section{(i) The QMD approach:}

The QMD model is based on molecular dynamics and hence is an n-body theory which simulates the heavy ion reactions between $30 \mathrm{~A} \cdot \mathrm{MeV}$ to $1 \mathrm{~A} \cdot \mathrm{GeV}$ on an event by event basis [10]. Here each nucleus is represented by a coherent state of the form $(\hbar=1)$

$$
\phi_{\alpha}\left(x_{1}, t\right)=\left(\frac{2}{\pi L}\right)^{3 / 4} e^{-\left(\vec{x}_{1}-\vec{x}_{\alpha}-\frac{\vec{p}_{\alpha} t}{m}\right)^{2} / 2 L} e^{i \vec{p}_{\alpha}\left(\vec{x}_{1}-\vec{x}_{\alpha}\right)} e^{-\frac{i p_{\alpha}^{2} t}{2 m}}
$$

The wave function has two time dependent parameter $x_{\alpha}, p_{\alpha}$. We fix the Gaussian width $(L)$ to $1.08 \mathrm{fm}^{3}$. In QMD calculations, nucleon $\alpha$ moves on a quasi-classical trajectory as obtained by a variation solution of the n-body Schrodinger equation:

$$
\begin{gathered}
\dot{\vec{x}}_{\alpha}=\frac{p_{\alpha}}{m}+\nabla_{p_{\alpha}} \sum_{\beta}<V_{\alpha \beta}\left(x_{\alpha}, x_{\beta}, p_{\alpha}, p_{\beta}\right)> \\
\dot{\vec{p}}_{\alpha}=-\nabla_{\vec{x}_{\alpha}} \sum_{\beta}<V_{\alpha \beta}\left(x_{\alpha}, x_{\beta}, p_{\alpha}, p_{\beta}\right)>
\end{gathered}
$$

Here $p_{\alpha}$ and $\mathrm{x}_{\alpha}$ are the centriods of the Gaussian wave functions in momentum and coordinate space which represent the nucleon $\alpha$. The potential has the form [3]

$$
<V_{\alpha \beta}\left(x_{\alpha}, x_{\beta}\right)>=\int d^{3} x_{1} d^{3} x_{2}<\phi_{\alpha} \phi_{\beta}\left|V\left(x_{1}, x_{2}\right)\right| \phi_{\alpha} \phi_{\beta}>
$$

In addition, the nucleons interact via stochastic elastic and inelastic NN collisions. In principle, our approach to find the fragments is independent of the algorithm which generates the phase space coordinates. Therefore, QMD may 
be replaced by any other model ( like simple molecular dynamics model [6], Boltzmann-Uhling-Uhlenbeck model etc. [11) which is able to generate the phase space coordinates of the particles. Due to its n-body nature, the QMD model is more appropriate to study the fragment formation in heavy ion collisions than one body models.

\section{(ii) A Survey of Heavy Ion Reaction:}

During the simulation of the reaction, we store the phase space coordinates of all nucleons at several time steps. As the QMD model simulates the time evolution of nucleons, the stored phase space distribution is that of nucleons only. Our basic assumption is that in nature that configuration is realized which gives the largest binding energy. Therefore, a method has to be adopted to group the nucleons in free nucleons and fragments. The nucleons within a fragment will be bound by some binding energy. In a very simple model, one could consider the nucleons being a part of the same fragment if their centriods are closer than some spatial distance $r_{\text {max }}$. This model is called minimum spanning tree [MST] method [3,?] . One generally takes $2 \leq r_{\max } \leq 4$. By definition, this method cannot address the fragment distribution during the violent phase of the reaction where whole nuclear matter is compressed and is confined to few fermis. The MST method at this time will give one single large fragment. More disturbing, the fragments detected by the MST method can contain nucleons with very large relative momenta. These fragments will be unstable and will decay after a while by fissioning or by emitting nucleons. To improve the model, a cut in momentum space has been also suggested recently by one of us and collaborators [7]. This cut (which limits the maximal allowed relative momentum of two nucleons in the same fragment) is quite effective in central collisions where most of the fragments are created during a reaction, but has no effect on the fragment distribution in peripheral collisions where the fragments are produced due to the decay of the spectator matter.

If one combines the cuts in momentum and in coordinate space to a binding energy cut, one sees that several groups of nucleons are indeed not fragments, but a group of unbound nucleons which are close in spatial space. One has to follow the reaction for a long time until this group of nucleons decays in light and heavy fragments which are well separated in the coordinate space and can be detected with the standard MST algorithm. The critical time is generally assumed to be about $300 \mathrm{fm} / \mathrm{c}$. 
To give the reader a more clear picture, we simulated the reaction $\mathrm{Au}-\mathrm{Au}$ at $600 \mathrm{MeV} / \mathrm{nucl}$. and at impact parameters of $\mathrm{b}=3$ and $8 \mathrm{fm}$, respectively, and display some key quantities in fig.1. The solid and dotted lines represent the reaction at 8 and $3 \mathrm{fm}$, respectively. The first row shows the evolution of mean density and of the collisions as a function of time. As expected, a higher density and collision number can be seen in central collisions compared to peripheral collisions. One also notices that the high reaction rate terminates at about $40-60 \mathrm{fm} / \mathrm{c}$. Afterwards, we observe only collisions of nucleons in the same fragment. The second row shows the evolution of spectator ( filled circle) and participant ( filled triangle) nucleons. A participant nucleon is defined as a nucleon which has undergone at least one collision. One sees that in central collisions $99 \%$ of nucleons have experienced a collision until $40 \mathrm{fm} / \mathrm{c}$. As a results the directed transverse flow saturates as early as $40-60 \mathrm{fm} / \mathrm{c}$. The fig. 1(e) displays the evolution of the size of the largest fragment $A^{\max }$ detected by the normal MST method with $r_{\max }=4 \mathrm{fm}$. We see one big fragment (consisting of 394 nucleons) at the time when the density is high. After about $120 \mathrm{fm} / \mathrm{c}$ we are able to find the "stable" fragment, which still decreases in size due to evaporation. Is this a realistic identification of the largest fragment? To answer this question, we applied a binding energy cut on the fragments detected in MST method. We first analyze the fragments with MST method and then pass all the fragments (with mass $\geq 3$ ) through an energy filter which recognizes a fragment only if it has at least a binding energy of $4 \mathrm{MeV} /$ nucl. Otherwise it considers the MST fragment as a set of free nucleons. This approach is labelled as $\mathrm{MST}^{\star}$. In both central and peripheral collisions, the largest fragment detected in MST is not a bound fragment at intermediate times. One gets properly bound fragment after about $120 \mathrm{fm} / \mathrm{c}$ after emitting the nucleons what lowers of course the binding energy. One should keep in mind that in peripheral collisions, one has two big (spectator) fragments and a fireball at mid-rapidity region without fragment.

\section{(iii) Simulated Annealing Clusterization Algorithm [SACA]:}

Our new approach can be summarized as follows. We assume that :

1 The nucleons from target and projectile are grouped into fragments (of any size) and into free nucleons.

2 Though the nucleons inside a fragment can interact with each other, they do not interact with the nucleons from other fragments or free nucleons.

3 That pattern of nucleons and fragments is realized in nature which gives the highest binding energy.

To avoid that at intermediate times too many fragments are assumed (which finally break apart), we employ in addition a binding energy check. In order to form a fragment, the considered group of nucleons has to have a minimal 
binding energy given by

$$
\zeta=\sum_{\alpha=1}^{N^{f}}\left[\sqrt{\left(\vec{p}_{\alpha}-\vec{P}_{N^{f}}^{c m}\right)^{2}+m_{\alpha}^{2}}-m_{\alpha}+\frac{1}{2} \sum_{\beta \neq \alpha}^{N^{f}} V_{\alpha \beta}\left(x_{\alpha}, x_{\beta}\right)\right]<L_{b e} \times N^{f},
$$

with $L_{b e}=-4.0 \mathrm{MeV}$ if $N^{f} \geq 3$ and $L_{b e}=0$ otherwise. In this equation, $N^{f}$ is the number of nucleons in a fragment, $\vec{P}_{N^{f}}^{c m}$ is the center-of-mass momentum of the fragment. The binding energy criteria will make sure that no loosely bound fragments are formed in our approach. In reality these loosely bound fragments are not stable and decay during the reaction. The problem is that we have to find the most bound configuration among a huge number of possible patterns ( composed of nucleons and fragments). In order to cope with this complicated problem, we employ the simulated annealing technique and hence this algorithm is dubbed as "Simulated Annealing Clusterization Algorithm (SACA)".

One is tempted to start the search for the most bound cluster configuration by an iterative minimization method (also known as neighborhood search or local search). In this method, starting from a given configuration a new one is constructed. The new configuration is accepted only if it lowers the binding energy. The drawback of this procedure is that it may terminate at a local minimum. To improve this limitation, several modification can be imagined [12]:

1. To execute the algorithm for a large number of the initial configurations. This will finally allow to reach the global minimum. This is very time consuming.

2. To use a algorithm which can jump over local minima and hence one can reach the global minima. This clearly depends strongly on the problem. Therefore its applications are limited.

3: To generalize the iterative method so that the transitions which yields a higher binding energy are always accepted. In addition, the transitions which yield a lower binding energy are also accepted with a certain probability. This algorithm is known as simulated annealing method [12. Its name is based on the fact that this algorithm is akin to the one used for cooling the solids. The simulated annealing method is a sequence of metropolis algorithms [13] with decreasing control parameter $\vartheta$. The control parameter $\vartheta$ can be interpreted as a "temperature". For each Metropoliscity at a given temperature, we perform a sequence of steps until the binding energy does not change anymore. Each step is executed as follows:

1: Given some initial configuration $\psi$ with energy $\zeta_{\psi}$, a new configuration $\varphi$ with energy $\zeta_{\varphi}$ is generated in the neighborhood of $\psi$ using a Monte-Carlo procedure.

2: Let the energy difference between $\psi$ and $\varphi$ is $\Delta \zeta=\zeta_{\varphi}-\zeta_{\psi}$. 
3.: If $\Delta \zeta$ is negative, the new configuration is always accepted. If $\Delta \zeta$ is positive, it is accepted with a probability $\exp (-\Delta \zeta / \vartheta)$. At the start, the control parameter $\vartheta$ is taken to be large enough for that most all attempted transitions are accepted. This is to overcome any kind of the local minima. After the binding energy remains constant, a gradual decrease in the control parameter $\vartheta$ is made and the Metropolis algorithm is repeated.

Note that there is no change in the coordinates of the nucleons. One should also note that employing this method does guaranty in the limit of infinite steps to reach the global minimum. Evidence that one reaches the ground state can be provided by obtaining the same fragment pattern for different starting configurations.

To start with, a random configuration $\psi$ ( which consist of fragments and free nucleons) is chosen. The total energy associated with $\psi$ configuration is given by

$$
\begin{aligned}
\zeta_{\psi} & =\sum_{\alpha=1}^{N_{1}^{f}}\left\{\sqrt{\left(\vec{p}_{\alpha}-\vec{P}_{N_{1}^{f}}^{c m}\right)^{2}+m_{\alpha}^{2}}-m_{\alpha}+\frac{1}{2} \sum_{\beta \neq \alpha}^{N_{1}^{f}} V_{\alpha \beta}\left(x_{\alpha}, x_{\beta}\right)\right\}_{1} \\
+ & \cdots \sum_{\alpha=1}^{N_{\nu}^{f}}\left\{\sqrt{\left(\vec{p}_{\alpha}-\vec{P}_{N_{\nu}^{f}}^{c m}\right)^{2}+m_{\alpha}^{2}}-m_{\alpha}+\frac{1}{2} \sum_{\beta \neq \alpha}^{N_{\nu}^{f}} V_{\alpha \beta}\left(x_{\alpha}, x_{\beta}\right)\right\}_{\nu} \\
+ & \sum_{\alpha=1}^{N_{\mu}^{f}}\left\{\sqrt{\left(\vec{p}_{\alpha}-\vec{P}_{N_{\mu}^{f}}^{c m}\right)^{2}+m_{\alpha}^{2}}-m_{\alpha}+\frac{1}{2} \sum_{\beta \neq \alpha}^{N_{\mu}^{f}} V_{\alpha \beta}\left(x_{\alpha}, x_{\beta}\right)\right\}_{\mu} \\
+ & +\sum_{\alpha=1}^{N_{n}^{f}}\left\{\sqrt{\left(\vec{p}_{\alpha}-\vec{P}_{N_{n}^{f}}^{c m}\right)^{2}+m_{\alpha}^{2}}-m_{\alpha}+\frac{1}{2} \sum_{\beta \neq \alpha}^{N_{n}^{f}} V_{\alpha \beta}\left(x_{\alpha}, x_{\beta}\right)\right\}_{n}
\end{aligned}
$$

Here $N_{\mu}^{f}$ is the number of nucleons in a fragment $\mu, \vec{P}_{N_{\mu}^{f}}^{c m}$ is the center of mass momentum of the fragment $\mu$ and $V_{\alpha \beta}\left(x_{\alpha}, x_{\beta}\right)$ is the interaction energy between nucleons $\alpha$ and $\beta$ in a given fragment $\mu$. Note that the total energy is the sum of the energies of individual fragments in their respective center of mass system. Therefore, $\zeta_{\psi}$ differs from the (conserved) total energy of the system because (i) the kinetic energies of fragments calculated in their center of masses and (ii) the interactions between fragments/free nucleons are neglected. At present a simple static interaction is implemented, but one can use the algorithm for arbitrary interactions.

A new configuration is generated using Monte-Carlo procedure by either a) transferring a nucleon from some randomly chosen fragment to another fragment or by b) setting a nucleon of a fragment free or c) absorbing a free nucleon into a fragment. Let the new configuration $\varphi$ be generated by transferring a nucleon from fragment $\nu$ to 
fragment $\mu$. Then the energy of new configuration $\varphi$ is given by:

$$
\begin{gathered}
\zeta_{\varphi}=\sum_{\alpha=1}^{N_{1}^{f}}\left\{\sqrt{\left(\vec{p}_{\alpha}-\vec{P}_{N_{1}^{f}}^{c m}\right)^{2}+m_{\alpha}^{2}}-m_{\alpha}+\frac{1}{2} \sum_{\beta \neq \alpha}^{N_{1}^{f}} V_{\alpha \beta}\left(x_{\alpha}, x_{\beta}\right)\right\}_{1} \\
+\cdots \sum_{\alpha=1}^{N_{\nu}^{f}-1}\left\{\sqrt{\left(\vec{p}_{\alpha}-\vec{P}_{N_{\nu}^{f}-1}^{c m}\right)^{2}+m_{\alpha}^{2}}-m_{\alpha}+\frac{1}{2} \sum_{\beta \neq \alpha}^{N_{\nu}^{f}-1} V_{\alpha \beta}\left(x_{\alpha}, x_{\beta}\right)\right\}_{\nu} \\
+\sum_{\alpha=1}^{N_{\mu}^{f}+1}\left\{\sqrt{\left(\vec{p}_{\alpha}-\vec{P}_{N_{\mu}^{f}+1}^{c m}\right)^{2}+m_{\alpha}^{2}}-m_{\alpha}+\frac{1}{2} \sum_{\beta \neq \alpha}^{N_{\mu}^{f}+1} V_{\alpha \beta}\left(x_{\alpha}, x_{\beta}\right)\right\}_{\mu} \\
+\cdots \sum_{\alpha=1}^{N_{n}^{f}}\left\{\sqrt{\left(\vec{p}_{\alpha}-\vec{P}_{N_{n}^{f}}^{c m}\right)^{2}+m_{\alpha}^{2}}-m_{\alpha}+\frac{1}{2} \sum_{\beta \neq \alpha}^{N_{n}^{f}} V_{\alpha \beta}\left(x_{\alpha}, x_{\beta}\right)\right\}_{n}
\end{gathered}
$$

Note that in this procedure, the individual energies of all fragments except for the donar fragment $(\nu)$ and the receptor fragment $(\mu)$ remain the same. The change in the energy from $\psi \longrightarrow \varphi$ is given by

$$
\Delta \zeta=\zeta_{\varphi}-\zeta_{\psi}
$$

Between the Metropolis algorithms, we cool the system by decreasing the control parameter $\vartheta$. A decrease in the temperature means that we narrow the energy difference which is accepted in a metropolis step. After many Metropolis steps, we should arrive at a minimum i.e. the most bound configuration. The problem is, however, that we usually arrive at a local minimum only. Between the local minimum, we find huge maxima. Let us give an example: Assume we have two fragments, but the most bound configuration would be one single fragment which combines both. Now each exchange of a single nucleons raises the binding energy and only the exchange of all nucleons at the same time lowers the total binding energy. This effect is well known in chemistry, where it is called Activation energy. In order to avoid this, we add, therefore, a second simulated annealing algorithm in which not anymore the nucleons are considered as the particles which are exchanged in each Metropolis step (like in the first simulated annealing), but the entities ( fragments or nucleons) obtained after the first step. This second stage of minimization is called fragment exchange procedure. This fragment exchange procedure is capable of overcoming any local minima.

Note that even in this second stage of the minimization, the free nucleons can be exchanged as before. The total energy associated with any configuration $\Psi$ during second stage of iterations is given by

$$
\zeta_{\Psi}=\left\{\sum_{\alpha=1}^{N_{S_{1}}}\left[\sqrt{\left(\vec{p}_{\alpha}-\vec{P}_{N_{S_{1}}}^{c m}\right)^{2}+m_{\alpha}^{2}}-m_{\alpha}+\frac{1}{2} \sum_{\beta \neq \alpha}^{N_{S_{1}}} V_{\alpha \beta}\left(x_{\alpha}, x_{\beta}\right)\right]\right\}_{1}
$$




$$
\begin{gathered}
+\cdots\left\{\sum_{\alpha=1}^{N_{S_{\nu}}}\left[\sqrt{\left(\vec{p}_{\alpha}-\vec{P}_{N_{S_{\nu}}}^{c m}\right)^{2}+m_{\alpha}^{2}}-m_{\alpha}+\frac{1}{2} \sum_{\beta \neq \alpha}^{N_{S_{\nu}}} V_{\alpha \beta}\left(x_{\alpha}, x_{\beta}\right)\right]\right\}_{\nu} \\
+\left\{\sum_{\alpha=1}^{N_{S_{\mu}}}\left[\sqrt{\left(\vec{p}_{\alpha}-\vec{P}_{N_{S_{\mu}}}^{c m}\right)^{2}+m_{\alpha}^{2}}-m_{\alpha}+\frac{1}{2} \sum_{\beta \neq \alpha}^{N_{S_{\mu}}} V_{\alpha \beta}\left(x_{\alpha}, x_{\beta}\right)\right]\right\}_{\mu} \\
+\cdots\left\{\sum_{\alpha=1}^{N_{S_{n}}}\left[\sqrt{\left(\vec{p}_{\alpha}-\vec{P}_{N_{S_{n}}}^{c m}\right)^{2}+m_{\alpha}^{2}}-m_{\alpha}+\frac{1}{2} \sum_{\beta \neq \alpha}^{N_{S_{n}}} V_{\alpha \beta}\left(x_{\alpha}, x_{\beta}\right)\right]\right\}_{n}
\end{gathered}
$$

Here $N_{S_{\mu}}=\sum_{i=1}^{N_{S_{\mu}}^{f}} N_{S_{\mu}}^{i}$ is the number of nucleons in a super-fragment $S_{\mu} . N_{S_{\mu}}^{i}$ is the number of nucleons in the i-th fragment contained in the super-fragment $S_{\mu}$ and $N_{S_{\mu}}^{f}$ is the number of pre-fragments contained in the super-fragment $S_{\mu}$. The $\vec{P}_{N_{S_{\mu}}}^{c m}$ is the center of mass momentum of the super fragment $S_{\mu}$ and $V_{\alpha \beta}\left(x_{\alpha}, x_{\beta}\right)$ is the interaction energy between nucleons $\alpha$ and $\beta$ in a given super-fragment. Note that now the particle $\alpha$ interacts with its fellow nucleons in the same pre-fragment and also with the nucleons of other pre-fragments which are contained in a new given super fragment $S_{\mu}$.

Now the new configuration is generated using Monte-Carlo procedure by either a) transferring a pre-fragment from some randomly chosen super-fragment to another super-fragment or by b) setting a pre-fragment free or c) absorbing a single isolated pre-fragment into a super-fragment. Let us suppose that a new configuration $\Phi$ is generated by transferring a pre-fragment $\mathbf{i}$ ( with mass $N_{S_{\nu}}^{i}$ ) from super-fragment $\nu$ to super-fragment $\mu$. The associated energy of new configuration $\Phi$ reads as :

$$
\begin{array}{r}
\zeta_{\Phi}=\left\{\sum_{\alpha=1}^{N_{S_{1}}}\left[\sqrt{\left(\vec{p}_{\alpha}-\vec{P}_{N_{S_{1}}}^{c m}\right)^{2}+m_{\alpha}^{2}}-m_{\alpha}+\frac{1}{2} \sum_{\beta \neq \alpha}^{N_{S_{1}}} V_{\alpha \beta}\left(x_{\alpha}, x_{\beta}\right)\right]\right\}_{1} \\
+\cdots\left\{\sum_{\alpha=1}^{N_{S_{\nu}}-N_{S_{\nu}}^{i}}\left[\sqrt{\left(\vec{p}_{\alpha}-\vec{P}_{N_{S_{\nu}}-N_{S_{\nu}}^{i}}^{c m}\right)^{2}+m_{\alpha}^{2}}-m_{\alpha}+\frac{1}{2} \sum_{\beta \neq \alpha}^{N_{S_{\nu}}-N_{S_{\nu}}^{i}} V_{\alpha \beta}\left(x_{\alpha}, x_{\beta}\right)\right]\right\}_{\nu} \\
+\left\{\sum _ { \alpha = 1 } ^ { N _ { S _ { \mu } } + N _ { S _ { \nu } } ^ { i } } \left[\sqrt{\left.\left(\vec{p}_{\alpha}-\vec{P}_{\left.N_{S_{\mu}}+N_{S_{\nu}}^{i}\right)^{2}+m_{\alpha}^{2}}^{c m}-m_{\alpha}+\frac{1}{2} \sum_{\beta \neq \alpha}^{N_{S_{\mu}}+N_{S_{\nu}}^{i}} V_{\alpha \beta}\left(x_{\alpha}, x_{\beta}\right)\right]\right\}_{\mu}}\right.\right. \\
+\cdots\left\{\sum_{\alpha=1}^{N_{S_{n}}}\left[\sqrt{\left(\vec{p}_{\alpha}-\vec{P}_{N_{S_{n}}}^{c m}\right)^{2}+m_{\alpha}^{2}}-m_{\alpha}+\frac{1}{2} \sum_{\beta \neq \alpha}^{N_{S_{n}}} V_{\alpha \beta}\left(x_{\alpha}, x_{\beta}\right)\right]\right\}_{n}
\end{array}
$$

The only difference between the particle and the fragment exchange procedure occurs for the bound nucleons. Now the bound nucleons cannot change their identity neither by being absorbed nor by becoming free. They will remain 
bound in a pre-fragment. The pre-fragment itself can change its identity by either getting transferred to a new super-fragment, or be set free. As in the first stage, we calculate the energy difference between the new and the old configurations $\Delta \zeta$ and the metropolis procedure is continued till the most favored configuration is obtained.

In summary, the actual procedure is as follows: We first simulate the nucleus-nucleus collision using the QMD model and store the phase-space coordinates of all nucleons at several time steps. At each stored time step, we apply the SACA to find the most bound configuration which consists of nucleons and fragments (of any size). For a faster convergence of the algorithm, any cluster decomposition irrespective whether it fulfills the binding energy check (eq.

5) or not is considered. Therefore, it is likely that several clusters may fail to fulfill eq. (5). At the end of the algorithm when the most bound configuration is found, we check the binding energy ( eq. 5) of each superfragment explicitly and mark all super-fragments violating this condition. The nucleons belonging to an 'inhibited' (marked) cluster are further on treated as free nucleons. The minimizing procedure of the simulated annealing mechanism is invoked again until a configuration is found where all fragments fulfill eq. (5). The heavy fragments are usually more bound than the lighter ones. We have carried out a detailed analysis and found that these are always the light fragments ( with masses 3 or 4 ) which at the end of the iterations are unbound or loosely bound.

In the following, we discuss the numerical realization of the algorithm and present a detailed analysis of the influence of different parameters used in simulated annealing method.

\section{Numerical Realization:}

The simulated annealing algorithm has several parameters to be determined : the initial and the final value of the control parameter $\vartheta$, the number of metropolis steps to be executed at a given value of control parameter (i. e. length of Markov chain), the decrease of the control parameter and the termination of the algorithm. This set of parameters is also referred as cooling schedule in the literature [12]. One needs to choose the following parameters explicitly:

1.: The initial value of the control parameter $\vartheta_{i}$. This will be referred as temperature.

2.: The final value of the control parameter $\vartheta_{f}$ [i.e. the termination procedure].

3.: The length of the Markov chain $M_{c h}$. 
4.: A rule to fix the decrement in the control parameter $\sigma$.

Following [12], we use a so called simple cooling scheme and present the analysis of our extensive tests made for the collisions of Au-Au at $600 \mathrm{MeV} /$ nucl. and at an impact parameter of $8 \mathrm{fm}$. We have also analyzed the results for the collisions of $\mathrm{Pb}-\mathrm{Pb}$ (central) and $\mathrm{Nb}-\mathrm{Nb}$ (central and peripheral). The results of our analysis are independent of the masses of the colliding nuclei and also of the impact parameter. For our analysis we chose a conservative set of the above parameters and then try to find an optimized set of the parameters which yields the shortest computational time. We use the following set of parameters if not stated otherwise:

The initial temperature $\vartheta_{i}$ is taken to be $4 \mathrm{MeV}$. The length of Markov chain is taken to be $70 \eta$. ; $\eta$ being the number of entities at the start of the minimization. After each markov chain $[=70 \eta]$, the temperature is decreased using a simple law :

$$
\vartheta_{i+1}=\sigma \cdot \vartheta_{i}
$$

with $\sigma=0.95$. Finally, the algorithm is terminated if there is no change in the binding energy for a large number of iterations $[=60 \eta]$. The details of each of these parameters will be presented the following paragraphs.

i The Initial Configuration: We have to choose a random initial distribution initially to evoke the simulated annealing minimization. In our procedure, we distribute the nucleons (of the two colliding nuclei) into few cells. The transfer of nucleons is allowed among these cells. Naturally, the final outcome should be independent of the number of cells we choose. In fig. 2, we present the outcome of a single QMD event when exposed to SACA with a different number of initial cells. The displayed reaction is of $\mathrm{Au}-\mathrm{Au}$ at $600 \mathrm{MeV} / \mathrm{nucl}$. and impact parameter of 8 fm. Here we vary the number of cells between two and 394 ( that is by treating each nucleon as a free particle). We see that the variation in the cell number does not affect the final fragment distribution. At zero fm/c, the simulated annealing method finds two nuclei ( i.e. the projectile and target) which shows the validity of the annealing method. One also notices that the binding energy of the system remains constant between $40 \mathrm{fm} / \mathrm{c}$ and $200 \mathrm{fm} / \mathrm{c}$. In other words, the most bound configuration found in SACA at $40 \mathrm{fm} / \mathrm{c}$ and $200 \mathrm{fm} / \mathrm{c}$ is approximately the same.

In fig. 3, we display the evolution of most bound configuration using the two extremes : 2 cells and all particles free at 0, 40, 120 and $200 \mathrm{fm} / \mathrm{c}$, respectively. Note that the high density phase is reached around 40 fm/c. Between 
120 and $200 \mathrm{fm} / \mathrm{c}$, one should not expect much change as the reaction is already finished and there is only some rearrangement of the nucleons in the fragments. In case of 2 cells ( two initial clusters) the algorithm first breaks each of the clusters into large number of free nucleons (because free nucleons have zero energy) and some light fragments. After several hundred thousands iterations, it starts rearranging the nucleons into bound fragments. It is interesting to note that after some initial differences, the evolution of most bound configuration is quite the same in both cases.

As stated in the algorithm section, we choose the new configuration ( $\varphi$ or $\Phi$ ) by transferring a nucleon/pre-fragment from one fragment/superfragment to another. These fragments are chosen by Monte-Carlo method. It would be interesting to study the effect of different Monte-Carlo procedures (applied in SACA) on the fragment distribution. The different Monte-Carlo procedures can be generated using different random numbers. In figure 4, we display the fragment distribution ( i.e. the largest fragment $\mathrm{A}^{\max }$, the number of free nucleons and of intermediate mass fragments IMF's $A \geq 5$ ) and the energy of the most bound configuration at three different times i.e. at zero fm/c, 40 $\mathrm{fm} / \mathrm{c}$ and $200 \mathrm{fm} / \mathrm{c}$, respectively, for the different iterations. We see, as it should be, a almost complete independence.

ii. The Initial Value $\vartheta_{i}$ : One of the key features of the Metropolis algorithm is that it also accepts the transitions which increases the cost function i.e. the energy. Therefore, the initial value $\vartheta_{i}$ should be such that the most of the attempted transitions are accepted during first iterations. In other words, $\exp \left(-\Delta \zeta / \vartheta_{i}\right) \sim 1$. A practical way to implement the sequence of $\vartheta_{i}$ 's is given by Johnson et.al. [14. There the average increase in the energy over large number of iterations $\bar{\Delta} \zeta$ is related with $\vartheta_{i}$ by

$$
\text { acceptance ratio } \quad \chi=\exp \left\{-\bar{\Delta} \zeta / \vartheta_{i}\right\}
$$

i.e.

$$
\vartheta_{i}=\frac{\bar{\Delta} \zeta}{\ln \left(\chi^{-1}\right)}
$$

Generally, the acceptance ratio $\chi$ should be close to 1 . The choice of the value $\vartheta_{i}$ (or the temperature) depends very strongly on the problem at hand. It should be kept in mind that a very large value of $\vartheta_{i}$ will lead to huge computational time whereas a very small value will lead to less attempts which are accepted by the Metropolis algorithm and consequently which may lead to a wrong final distribution. In fig. 5, we show the same reaction as reported in fig. 1-4, but at different values of initial temperature $\vartheta_{i}$. Here the other parameters are kept unchanged. The variation 
of $\vartheta_{i}$ between $1 \mathrm{MeV}$ and $500 \mathrm{MeV}$ has no effect on the fragment distribution at zero $\mathrm{fm} / \mathrm{c}$. We have just two gold nuclei initially. On contrary, one can see some differences at $40 \mathrm{fm} / \mathrm{c}$. A very small value of $\vartheta_{i}(\leq 3-4 \mathrm{MeV})$ leads to a heavier $\mathrm{A}^{\max }(95)$ compared to the average $\mathrm{A}^{\max }(\approx 42)$ and as a result fewer IMF's and nucleons are emitted. Similar conclusions can be drawn at $200 \mathrm{fm} / \mathrm{c}$. A very low value of $\vartheta_{i}$ apparently freezes the initial configuration. The results are more stable for $\vartheta_{i} \geq 3 \mathrm{MeV}$. Therefore, we choose the $\vartheta_{i}=5 \mathrm{MeV}$.

In fig.6, we display the evolution of the most bound configuration in Au-Au reactions using $\vartheta_{i}=5 \mathrm{MeV}$ and $500 \mathrm{MeV}$. We see that when one iterates the reaction with very large initial temperature $(=500 \mathrm{MeV})$, almost all attempted transitions are accepted in the Metropolis algorithm. With a moderate value of the temperature $\vartheta_{i}=5$ $\mathrm{MeV}$, only some selected configurations are accepted. The minimization of the energy with $\vartheta_{i}=500 \mathrm{MeV}$ results in the vibration around the same fragments for very long time. An (unnecessary) large value of $\vartheta_{i}$ does not help to establish an early equilibrium. In contrary, one needs huge computation time (in terms of iterations) to find the most bound configuration. The same can be achieved with moderate value of $\vartheta_{i}=5 \mathrm{MeV}$ with far less costs.

iii. The Length of the Markov Chain: $M_{c h}$ : Here we fix the control parameter $\vartheta$ and execute the algorithm for a fixed number of Metropolis steps. We construct a sequence of fragment configurations $\mathrm{Q}=\{\psi, \varphi, \Psi, \ldots . ., \Phi\}$. One should note that here we have an initial configuration $\psi$ and new configuration $\varphi(=\psi+1)$ is generated by a random matrix. Thus, the number of iterations, and hence the length of Markov chain should be long enough to ensure an equilibrium. In fig. 7, we show the results with Markov chains of different length. The length of the markov chain $\eta$ is displayed in the units of the total number of the nucleons ( prefragments) present at the beginning of the minimization. After about $40 \eta$ the results are quite stable. For a smaller values of $\eta$, there are fluctuations in the results and in addition, SACA overestimates the size of $\mathrm{A}^{\max }$ and underestimates consequently the IMF's production. We fix the length of the markov chain $\eta=40$. The effect of different $\mathrm{M}_{c h}$ 's on the evolution of the most bound configuration is shown in fig. 8 where the evolution of the fragment's multiplicity is plotted as a function of the iterations for two values of Markov's chains i.e for $M_{c h}=40 \eta$ and $450 \eta$, respectively. Note that the number of iterations is $=\eta$ times the number of temperature steps. We see that the initial evolution is quite the same in both cases, but a smaller value of $M_{c h}$ needs less iterations than a longer one to arrive at same final value. This is easy to understand. The main aim of iterating over large number of iterations with same $\vartheta$ is to establish the quasi-equilibrium. Once an equilibrium is 
established, there will be no further improvements in the cost at same $\vartheta$, therefore, a smaller value of $M_{c h}$ leads to same result as that with largest $M_{c h}$.

iv. Decrement in the Control Parameter: The minimization is started with a relative large temperature $\vartheta_{i}$. Then the temperature is decreased in steps after a quasi-equilibrium is established for each temperature. Apparently, a larger decrement in the temperature will lead the defect to be frozen i.e. any configuration which may or may not be the most bound can freeze whereas a very small decrement will need huge computational time. The decrement should be in such a way that the length of the Markov chain $M_{c h}$ is as small as possible and thus after a new change of control parameter $\vartheta$, the quasi-equilibrium should be re-established as soon as possible. We here follow the simple rule for the decrement factor $\sigma$.

$$
\vartheta_{i+1}=\sigma \cdot \vartheta_{i}
$$

The value of $\sigma$ varies in the literature between 0.5 to 0.95 [12], [15], [14], 16]. The effect of different decrement factors $\sigma$ is displayed in fig. 9. Here all other parameters are kept the same as discussed at the beginning. One can see that a very small value of $\sigma$ overestimates the size of $A^{\max }$ and underestimates the IMF production. We fix the value of $\sigma$ to 0.85 . The comparison of two simulations resulting from the decrement factor $\sigma=0.85$ and 0.98 is displayed in fig. 10. Here we see that the two different values gives the same cooling result but for the larger value of $\sigma$ many more iterations are necessary.

iii. The Final Value $\vartheta_{f}$ : The termination procedure used in the literature varies from problem to problem and also from author to author. We fix the termination by two different controls.

1. Either we stop the calculations if the control parameter $\vartheta$ has reached a very small value where no further transition can be expected. For the present calculation, we take $\vartheta_{f}=10^{-10} \mathrm{MeV}$.

2. Or we terminate the algorithm if there is no change in the configuration over a large number of attempted iterations.. Following the rule used to fix the length of the Markov chain, we choose the length for termination $l_{t e r m}$ in terms of $\eta$ which represent the number of iterations in the units of $M_{c h}$. In fig. 11, we display the effect of the variation in $l_{\text {term }}$ on the fragment distribution. We find that different termination lengths do not affect the results. The effect of $l_{\text {term }}$ on the evolution of the most bound configurations is shown in fig. 12 where the evolution as a function of iterations is displayed for two values of $l_{\text {term }}$. i.e for $l_{\text {term }}=5 \eta$ and $120 \eta$, respectively. The different 
termination values have a very small effect on the fragment structure. Therefore, we fix $l_{\text {term }}$ to $35 \eta$.

In above paragraphs, we have discussed in detail the influence of different choices of the parameters which determine the simulated annealing method. One should note that once these parameters are chosen, the simulated annealing method is completely determined and it is a complete self-iterative method.

In the further discussion, the set with conservative parameters ( i.e. with $\vartheta_{i}=500 \mathrm{MeV}, M_{c h}=450 \eta, \sigma=0.98$ and $\left.l_{\text {term }}=120 \eta\right)$ is called as $\mathrm{S}_{l}$ whereas the set with the most economical parameters ( i.e. with $\vartheta_{i}=5 \mathrm{MeV}, M_{c h}=40 \eta$, $\sigma=0.85$ and $\left.l_{\text {term }}=35 \eta\right)$ is called as $\mathrm{S}_{e c}$.

The crucial test of the algorithm is its application to a single nucleus in its ground state. In principle one should get a single nucleus at the end. But the results can be different in reality. To see the importance of nucleon and fragment exchange procedured in SACA, we first turned off the fragment exchange part of the algorithm ( i.e. 2nd stage of the algorithm). As expected, the transfer of single nucleons terminates in local minima and as a result, one finds several fragments in the ground state of a nucleus. Naturally, the global minima is a single nucleus. The energy gain in a single nucleon transfer is not enough to overcome the huge energy barrier. This energy barrier can be overcome only by allowing the collective transfer of the nucleons i.e. of fragment as such. When we turned on the fragment exchange procedure of the algorithm, we could overcome the local minima and we find single nucleus as most bound configuration. In fig. 13, we show the evolution of the fragments as a function of the iterations for different single nuclei ${ }^{20} \mathrm{Ne},{ }^{40} \mathrm{Ca},{ }^{93} \mathrm{Nb}$ and ${ }^{208} \mathrm{~Pb}$, respectively using $S_{e c}$. Starting points are the (ground) state nuclei as generated by the QMD. In all cases, the SACA finds the single nucleus at the end of the iterations as it should. One also notices that the lighter nuclei need less iterations to find the most bound configuration. We find $\approx 8,000,12,000,62,000$ and 280, 000 iterations are necessary to find the ground state for ${ }^{20} \mathrm{Ne},{ }^{40} \mathrm{Ca},{ }^{93} \mathrm{Nb}$ and ${ }^{208} \mathrm{~Pb}$, respectively. One also notices that the increase in the number of necessary iterations is not a linear function of the masses of nuclei. The energy of the configurations is displayed in fig.14. We notice that one has a positive energy at the beginning which is decreased by breaking the cells into large number of nucleons/fragments. After a large number of iterations, one finally reaches the most bound configuration.

In fig. 15, we display the multiplicity (averaged over 20 events) of different fragments obtained using $S_{l}$ and $S_{c e}$, 
respectively. Here Au-Au collision is carried out at impact parameter of $8 \mathrm{fm}$. We see that both sets of parameters give a similar evolution of the reaction. One should note that the minimization with $S_{e c}$ needs much less computing time as compared to $S_{l}$. Our algorithm is able to detect the fragment distribution as early as $50-60$ fm/c. From fig. 1 , one notices that the density is maximum at this time. This very early identification of fragments in SACA is very promising because it means that the fragments may give insight into hot and dense nuclear matter.

The annealing algorithm can be made faster if some pre-information is feed into the algorithm. Naturally, the nucleons which are very far away in spatial or in momentum space will not lower the energy if one combines them as fragment. We applied a cut in spatial and in momentum space to sort out those distant nucleons. We took a minimal spatial distance between two nucleons of $10 \mathrm{fm}$ and a relative momentum of $200 \mathrm{MeV} / \mathrm{c}$. In other words, we

first break the whole system into fragments using these conditions and each of these fragments are then subjected to SACA. We found that the results are the same as before but the algorithm is about 10 times more faster.

\section{Summary:}

Summarizing, based on the simulated annealing method, we have presented the details of a new algorithm developed to study multifragmentation of heavy ion collisions. We have carried out an extensive survey of the different parameters which are crucial for the success of the method. Based on our calculations, a set of parameters is suggested for the algorithm which makes the algorithm very fast and accurate. This algorithm can detect the fragments as early as 40-60 fm/c i.e. at time when density is relative high and the interactions between fragments are still going on. It can not only give insight into the hot and dense nuclear matter, but at the same time makes it possible to apply the full in-medium G-matrix approach 17 to study the multifragmentation which was not possible due to the emission of nucleons after $70-80 \mathrm{fm} / \mathrm{c}$ [18]. A brief outcome of the results was presented in ref. 19] and a detailed physical interpretation of our results for various reactions will be presented elsewhere [20]. The algorithm is very general and may serve for every problem in which the most bound configuration has to be found.

One of us (RKP) appreciates the warm hospitality of SUBATECH, Ecole des Mines de Nantes, Nantes, France 
where this work was done. This work is supported by the CNRS and Ministry of Industry, Government of France. 


\section{Figure Captions:}

Fig. 1. Evolution of Au-Au collisions at incident energy of $600 \mathrm{MeV} /$ nucl. using a soft equation of state. The results at $\mathrm{b}=3$ and $8 \mathrm{fm}$ are displayed, respectively, by dotted and solid lines. Fig. 1(a) displays the mean density whereas the rate of collision is shown in Fig. 1(b). The evolution of the spectators ( filled circle) and the participants (filled triangle) is are presented in Fig. 1(c). Fig. 1(d) shows the time evolution of the transverse flow of the nucleons. Here, we do not consider the formation of fragments. The evolution of the largest mass $A^{\text {max }}$ formed within MST and MST with binding energy check $\left(\mathrm{MST}^{\star}\right)$ are displayed in fig. 1(e) and Fig. 1(f), respectively.

Fig. 2. The heaviest fragmnent $A^{\max }$, the emitted nucleons, the multiplicity of fragments with mass $\mathrm{A} \geq 5$ and the total energy associated with the configuration is displayed as a function of the cell number. Here the results at 0 , 40 and $200 \mathrm{fm} / \mathrm{c}$ are represented, respectively, by filled circle, open square and filled triangle. The displayed results are for a single event generated using QMD model.

Fig. 3. The evolution of the most bound configuration as a function of the iterations. Here we display the results at four times i.e. at 0, 40,120 and $200 \mathrm{fm} / \mathrm{c}$, respectively. The solid and dotted lines represents the results obtained with cells $=2$ and and 394, respectively.

Fig. 4 Same as fig. 2, but as a function of Monte-Carlo procedures.

Fig. 5 Same as fig. 4, but as a function of the initial temperature $\vartheta_{i}$. Here the arrow shows the value of the parameter chosen for the optimized set of parameters.

Fig. 6 Same as fig. 3, but with temperature $\vartheta_{i}=5 \mathrm{MeV}$ ( solid line) and $500 \mathrm{MeV}$ ( dotted line), respectively.

Fig. 7 Same as fig. 5, but as a function of the length of the Markov chain $M_{c h}$.

Fig. 8 Same as fig.3, but with $M_{c h}=40 \eta$ and $450 \eta$, respectively. 
Fig. 9 Same as fig. 5, but as a function of the decrement factor $\sigma$.

Fig. 10 Same as fig. 3, but with $\sigma=0.85$ and 0.98 , respectively.

Fig. 11 Same as fig. 5, but as a function of termination length $l_{\text {term }}$.

Fig. 12 Same as fig. 3, but with $l_{\text {term }}=35 \eta$ and $120 \eta$.

Fig. 13 Same as fig. 3, but the evolution of single nuclei $N e, C a, N b$ and $P b$, respectively.

Fig. 14 Same as fig. 13, but the energy of the system as a function of the iterations.

Fig. 15 The time evolution of the $\mathrm{Au}-\mathrm{Au}$ at $600 \mathrm{MeV} / \mathrm{nucl}$ and at an impact parameter of $8 \mathrm{fm}$ using a soft equation of the state. Here we display the results which are averaged over 20 events. The results obtained using $S_{l}$ and $S_{e c}$ are shown, respectively, by the filled circles and open squares, respectively. 
[1] M. B. Tsang et al., Phys. Rev. Letts 71, 1502 (1993); M. Begemann-Blaich et al., Phys. Rev. C 48, 610 (1993); J. Hubele et al., Phys. Rev. C 46, R1577 (1992); J. Hubele et al., Z. Phys. A340, 263 (1991); L. Phair et al., Phys. Letts. B 285, 10 (1992); W. Reisdorf et.al, Nucl. Phys. A 612493 (1997); A. Schuttauf et.al, Nucl. Phys. A607, 457 (1997).

[2] R.K. Puri et.al, Nucl. Phys. A 575, 733 (1994)

[3] J. Aichelin, Phys. Rep. 202, 233 (1991).

[4] R. K. Puri, C. Hartnack and J. Aichelin, Phys. Rev. C 54, R28 (1996).

[5] P. B. Gossiaux, R. Puri, C. Hartnack and J. Aichelin, Nucl. Phys. A 619, 379 (1997).

[6] C. Dorso and J. Randrup, Phys. Letts. B 301, 328 (1993).

[7] S. Kumar and R.K. Puri, Phys. Rev. C58,320 (1998); R.K. Puri and S. Kumar, Phys. Rev. C 57,2744 (1998); S. Kumar and R.K. Puri, Phys. Rev. C -in press (1998);S. Kumar, R.K. Puri and J. Aichelin, Phys. Rev. C (1998) in press.

[8] J.B. Garcia and C. Cerruti, Nucl. Phys. A578, 597 (1994).

[9] R.K. Gupta, S. Singh, R.K. Puri, and W. Schied, Phys. Rev. C47, 561 (1993).

[10] Ch. Hartnack at.al., Eur. Phys. J. A 1, 151 (1998)

[11] W. Bauer, G.F. Bertsch and S . DasGupta Phys. Rev. Letts. 58, 863 (1987).

[12] P.J. M. Laarhoven, and E.H. L. Aarts, Simulated Annealing: theory and applications ( Reidel, Dordrecht, 1987).

[13] N. Metropolis, A.W. Rosenblut, M.N. Rosenblut, A.H. Teller, and E. Teller, J. Chem Phys. 21,1087(1953).

[14] D.S. Johnson, C.R. Aragon, L.A. McGeoch, and C. Scheven, AT and T Bell Laboratories, preprints, 1987.

[15] S. Kirkpatrick, J. Stat. Phys, 34, 975 (1984).

[16] S. Nahar, S. Sahni, and E. Shragowitz, in "proceedings of 22nd Des. Automation conference, Las Vegas,USA,748 (1985).

[17] J. Jaenicke, J. Aichelin, N. Ohtsuka, R. Linden, and A. Faessler, Nucl. Phys. A 536, 201 (1992); A. Bohnet, N. Ohtsuka, J. Aichelin, R. Linden, and A. Faessler, Nucl. Phys. A494, 349 (1989).

[18] V. Ramillein et.al, Nucl. Phys. A 587,802 (1995).

[19] R.K. Puri, Ch. Hartnack and J. Aichelin, Phys. Rev. C54, R28 (1996); P.B. Gossiaux, R.K. Puri, Ch. Hartnack, and J. Aichelin, Nucl. Phys. A619, 379 (1997).

[20] R.K. Puri, Ch. Hartnack and J. Aichelin, under preparation. 


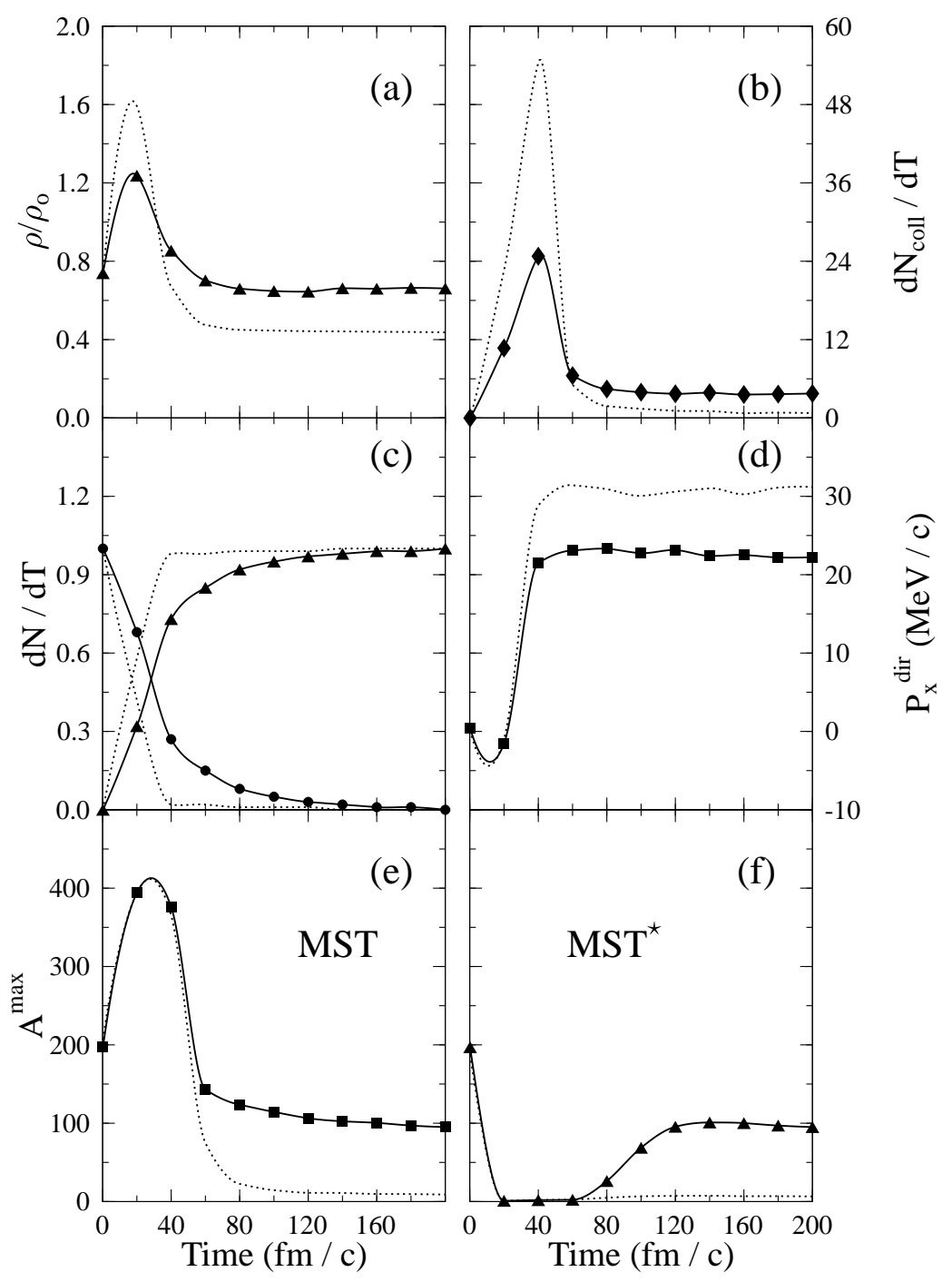

Fig. 1 


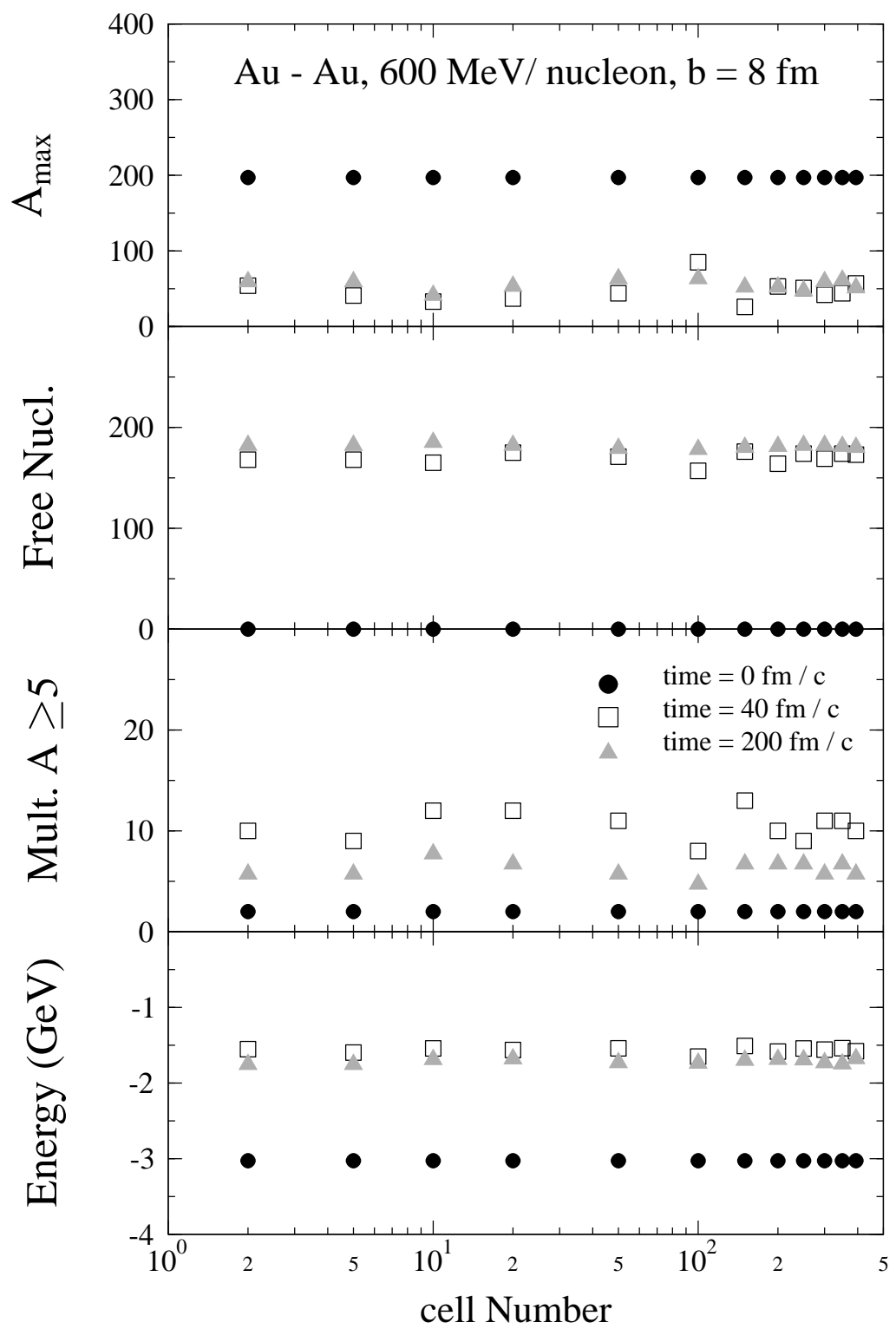

Fig. 2 

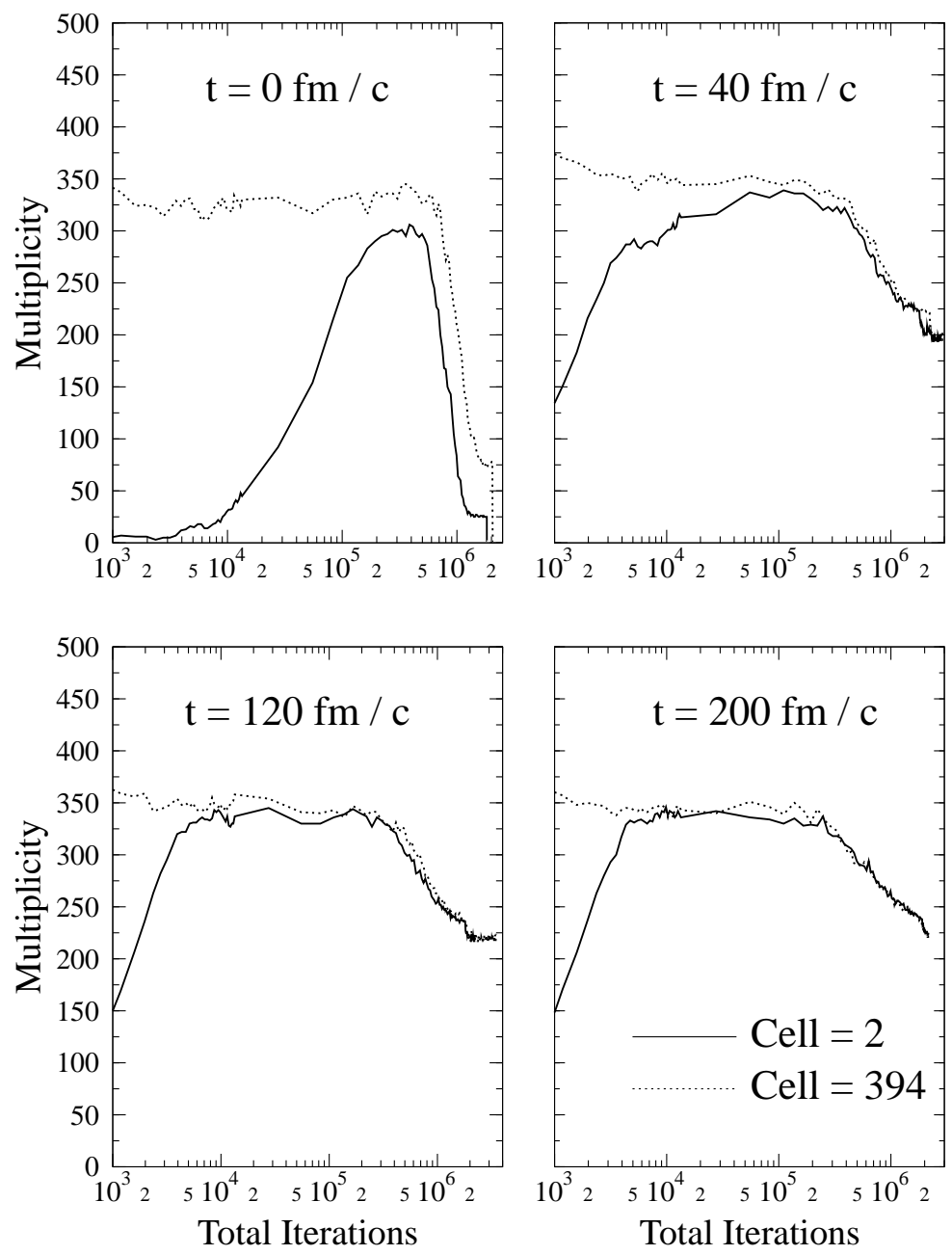

Fig. 3 


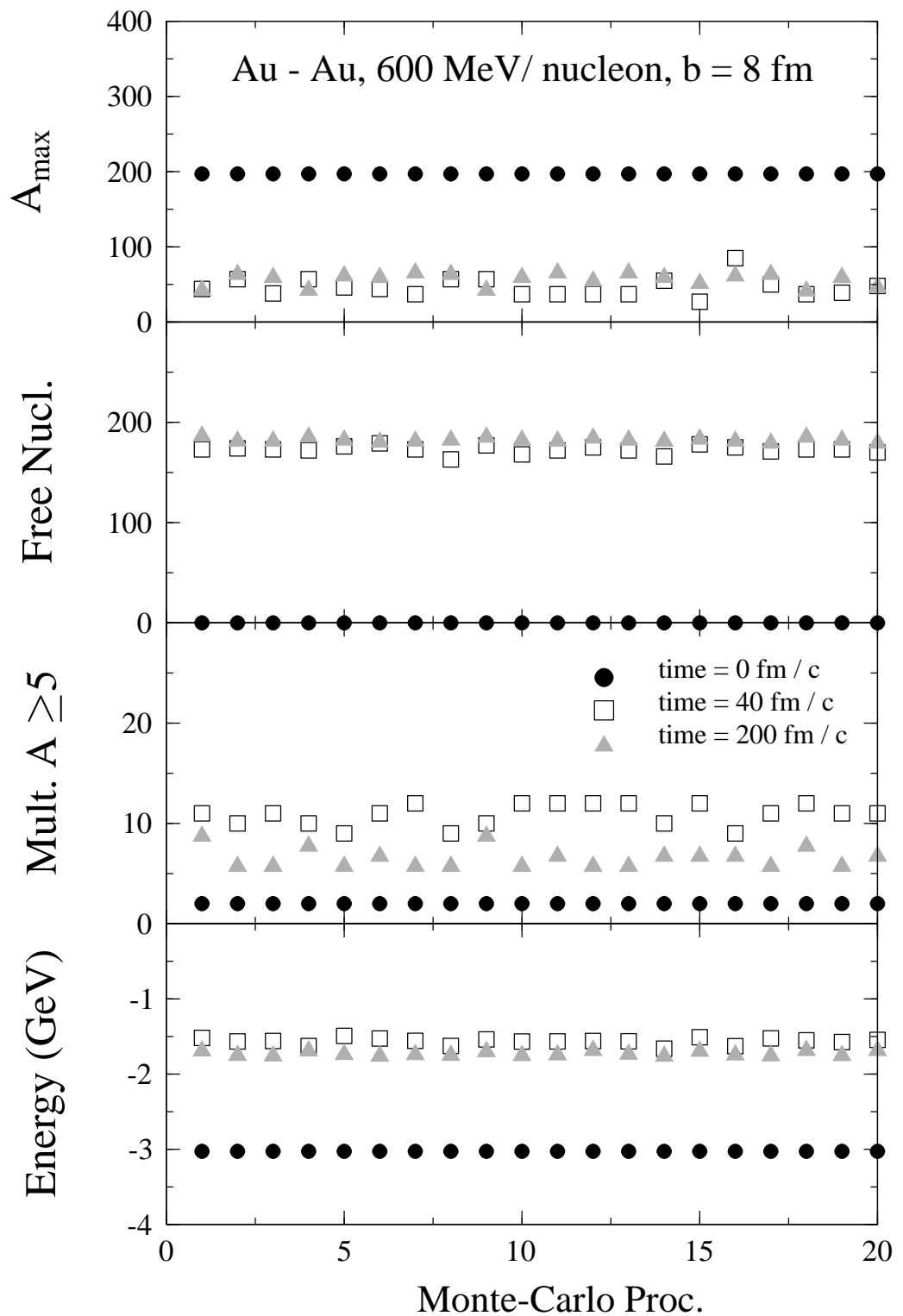

Fig. 4 


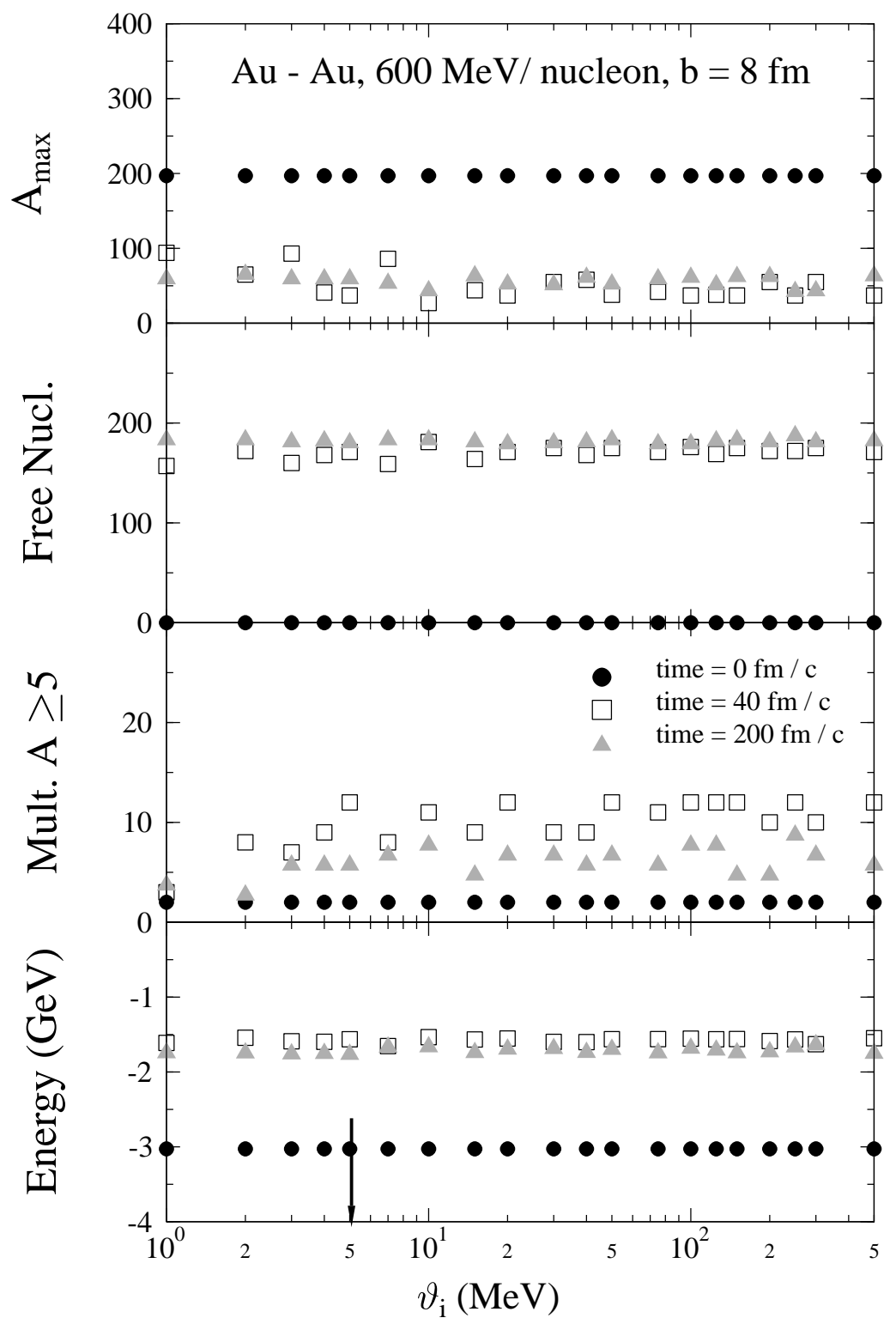

Fig. 5 

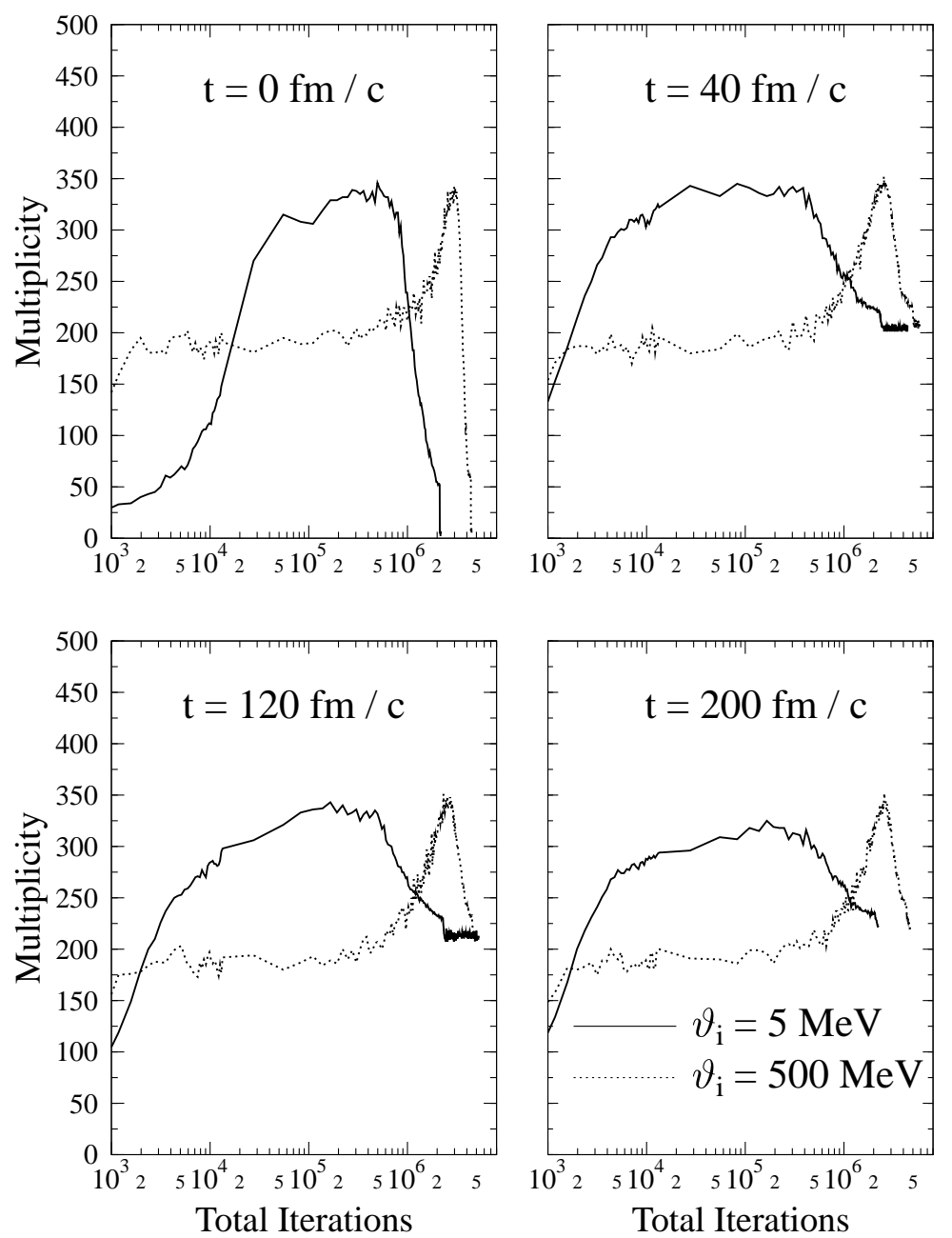

Fig. 6 


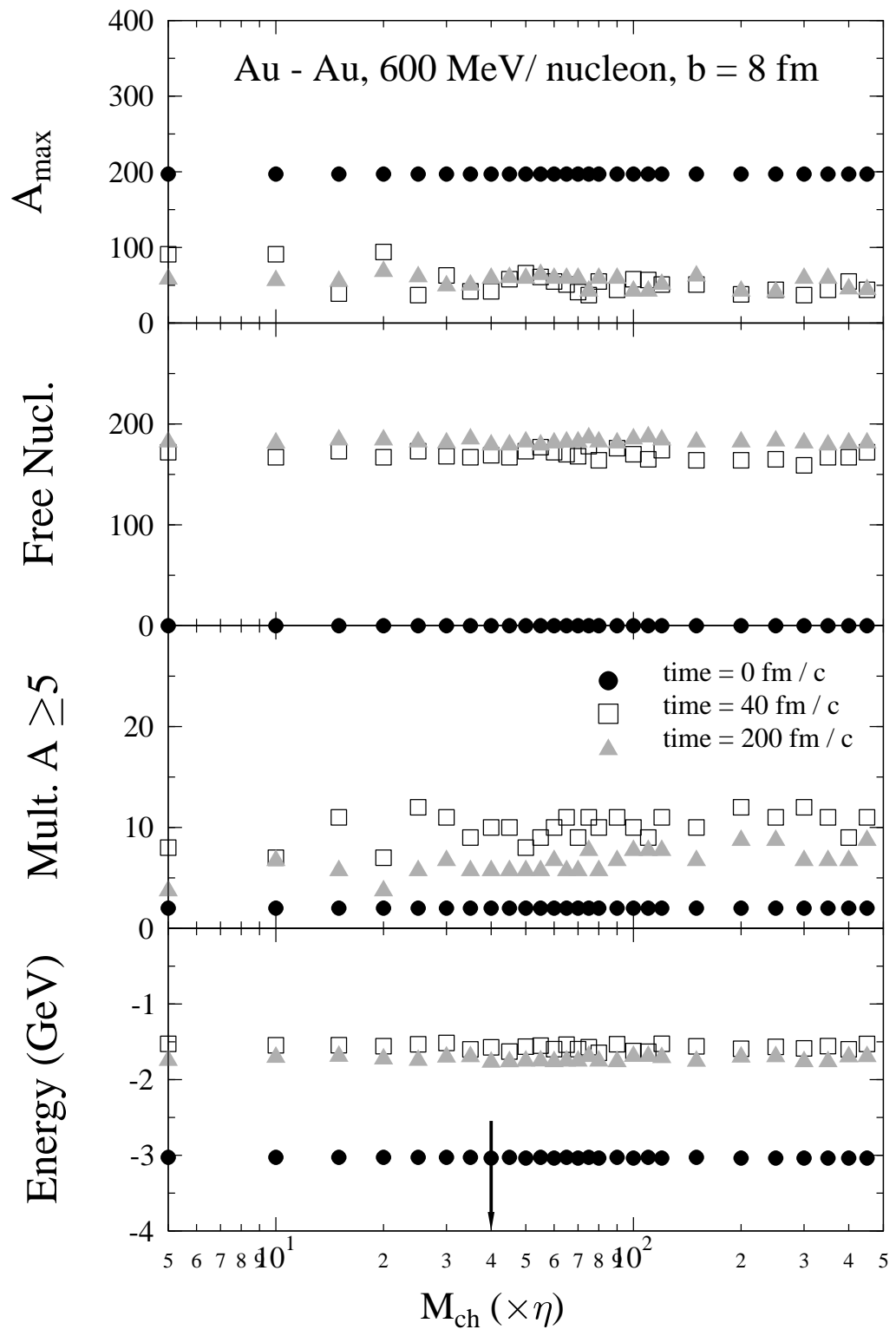

Fig. 7 

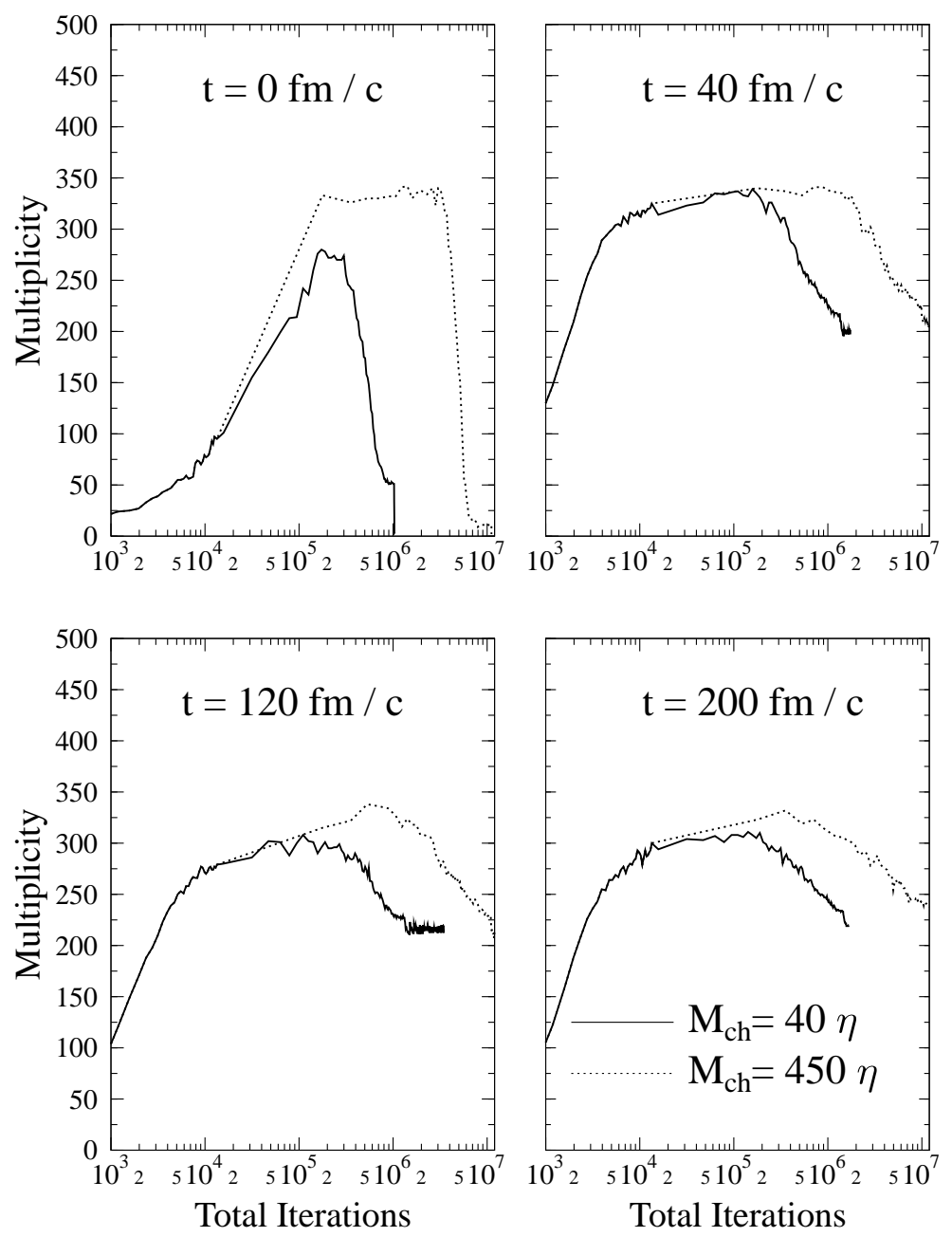

Fig. 8 


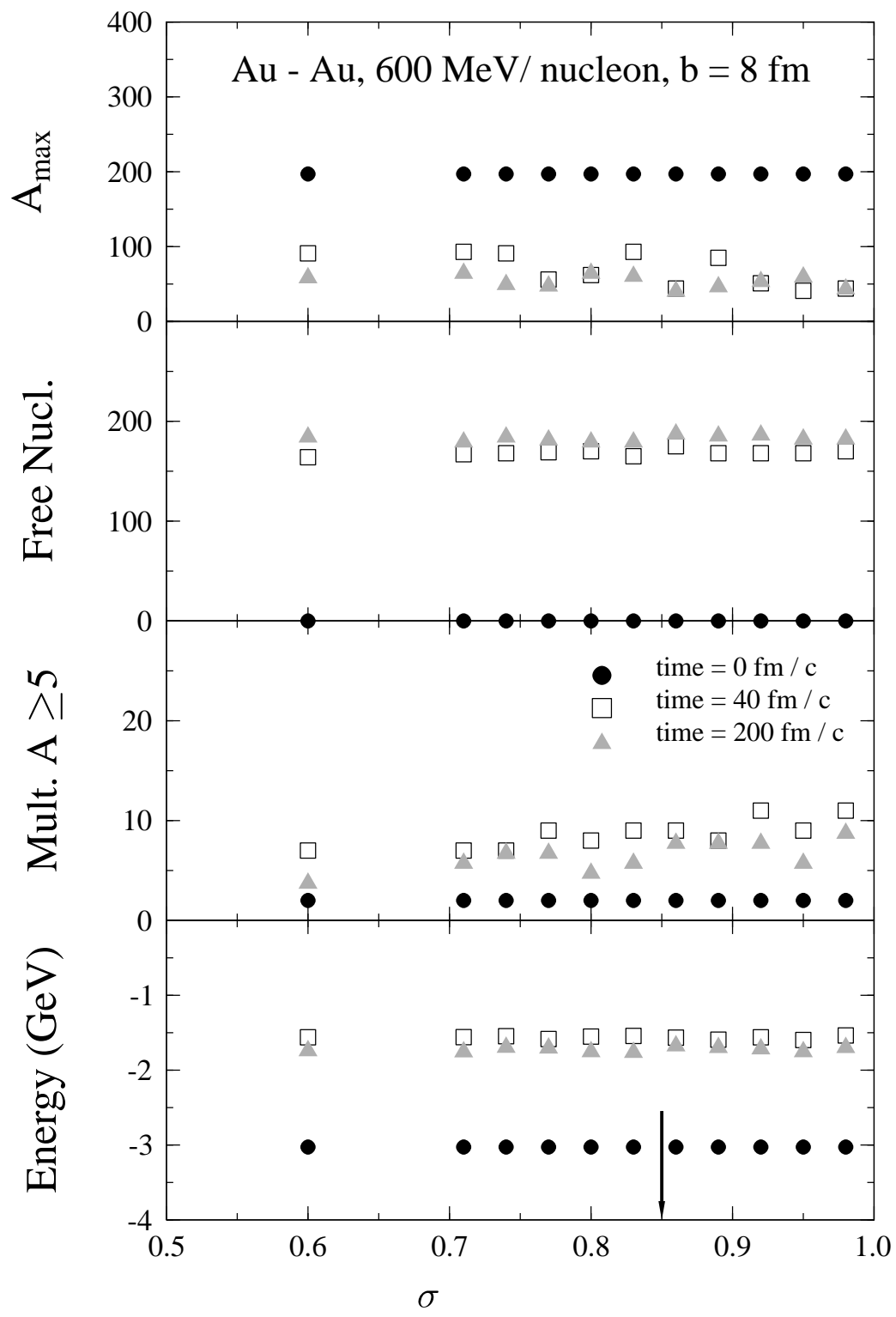

Fig. 9 

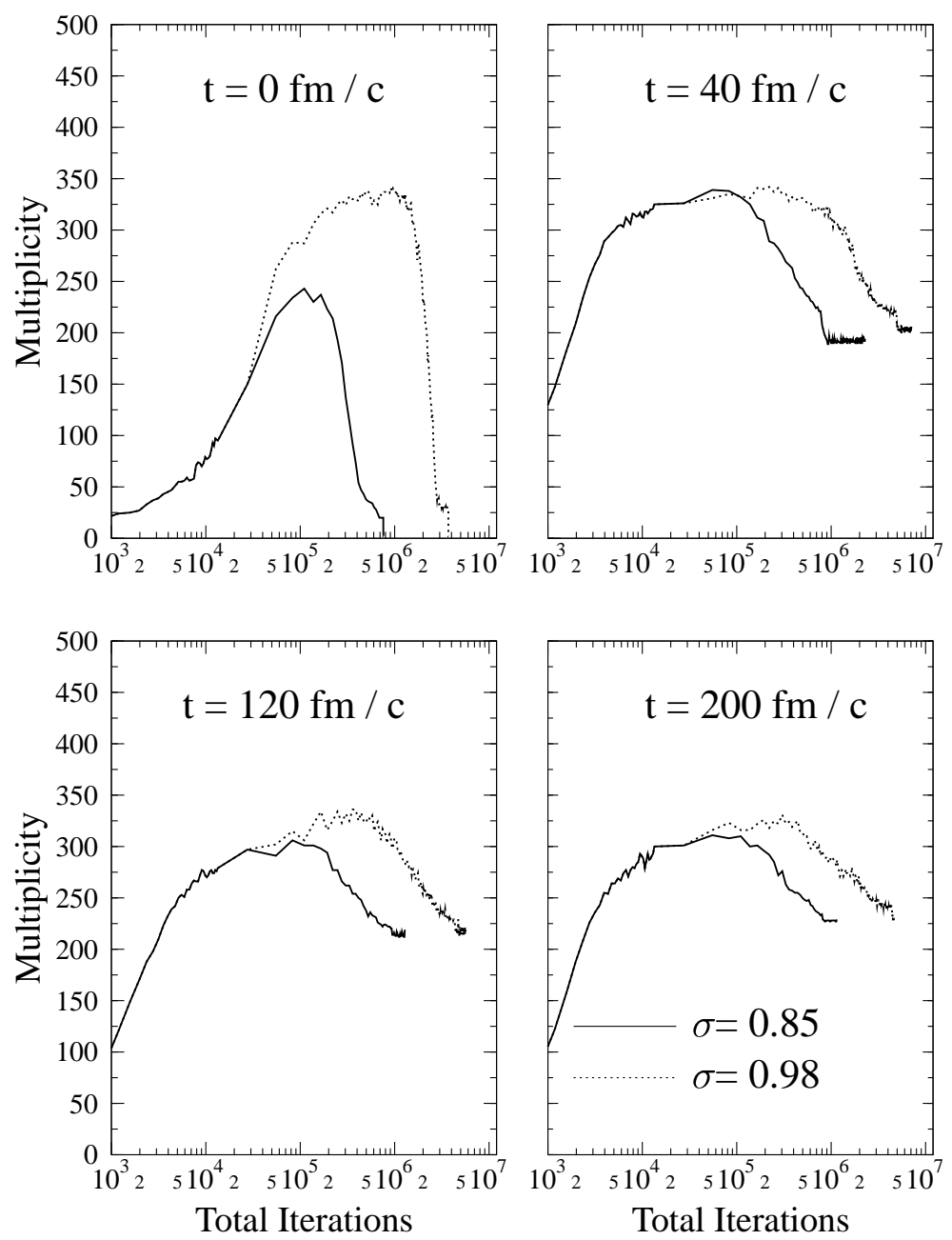

Fig. 10 


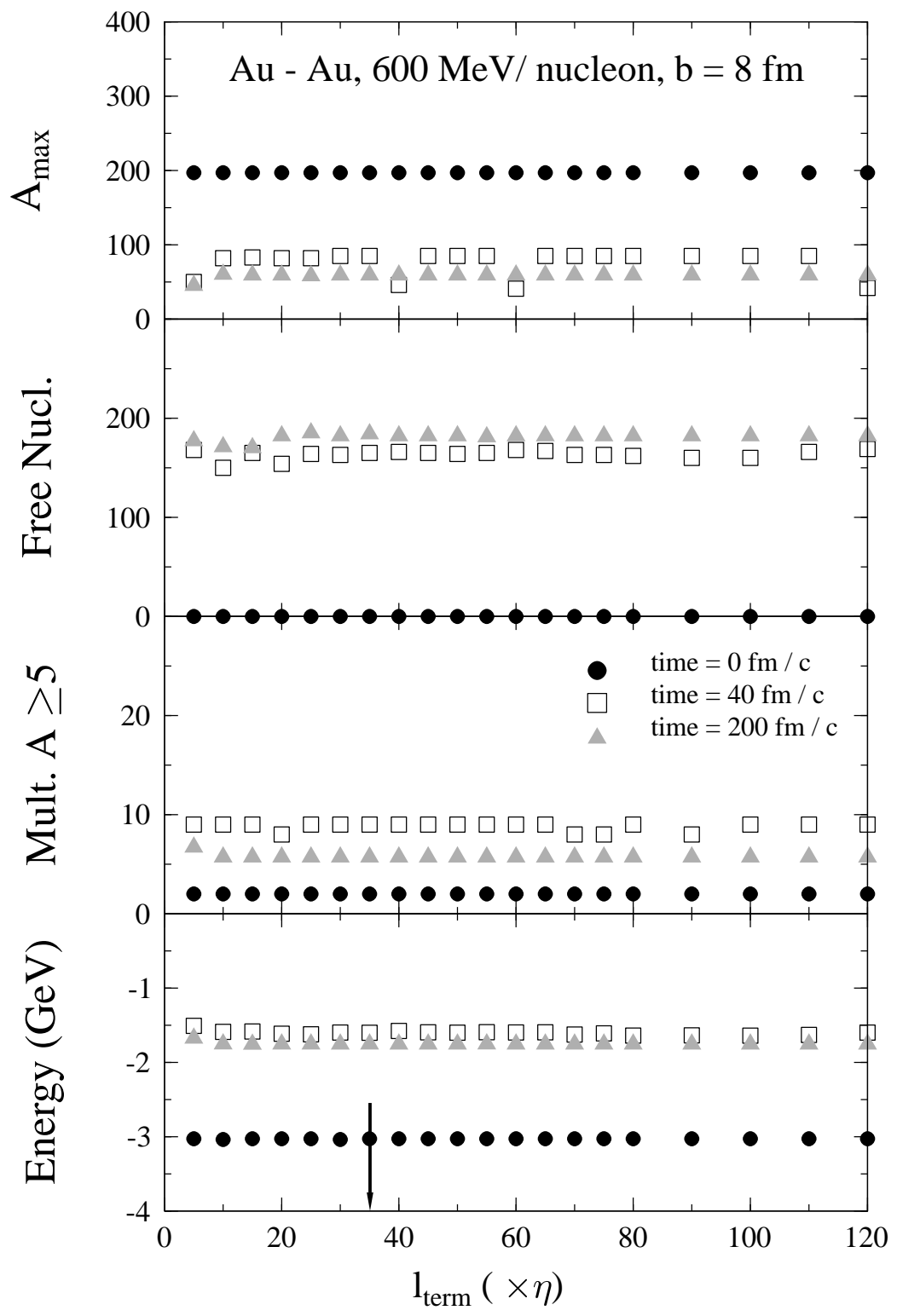

Fig.11 

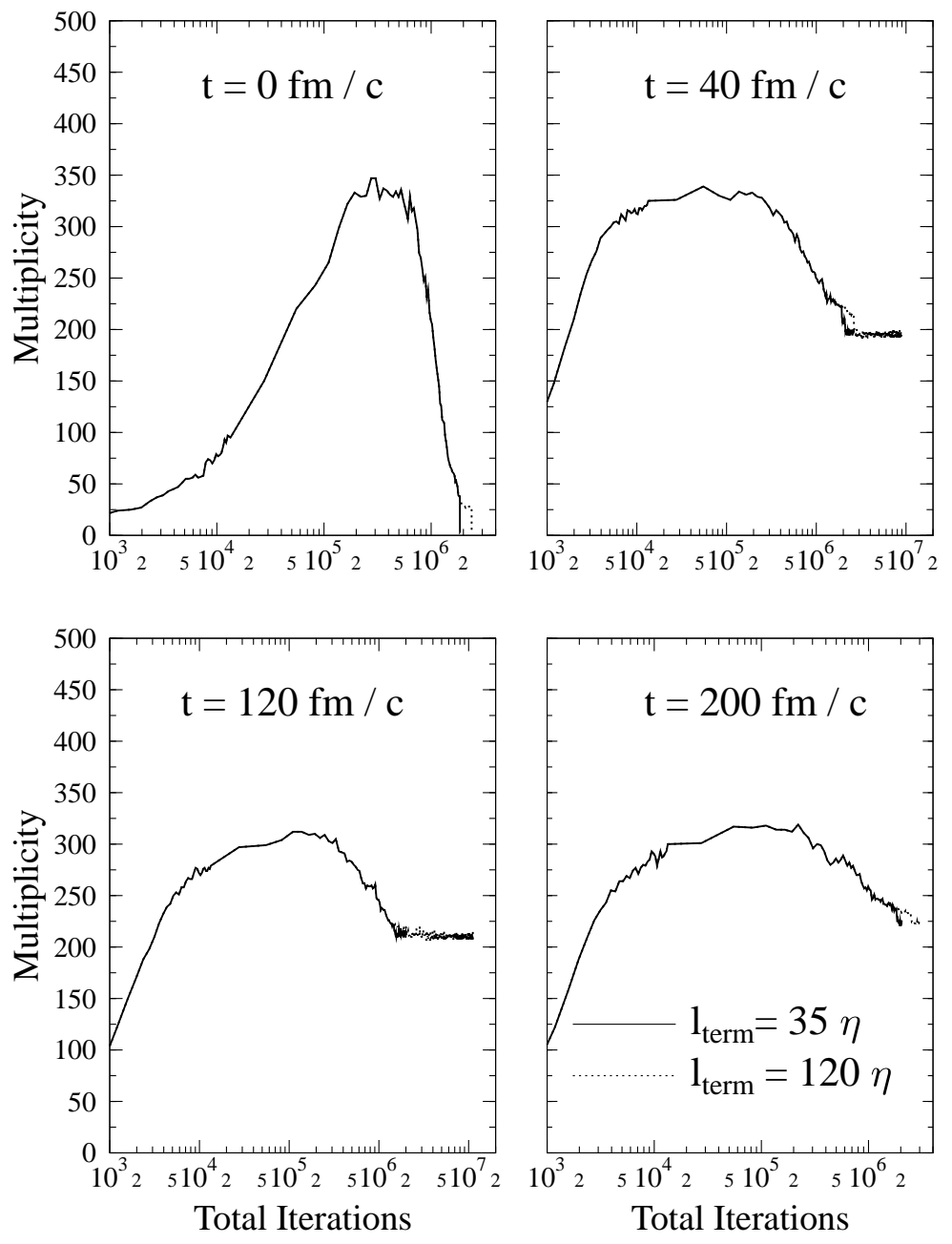

Fig. 12 

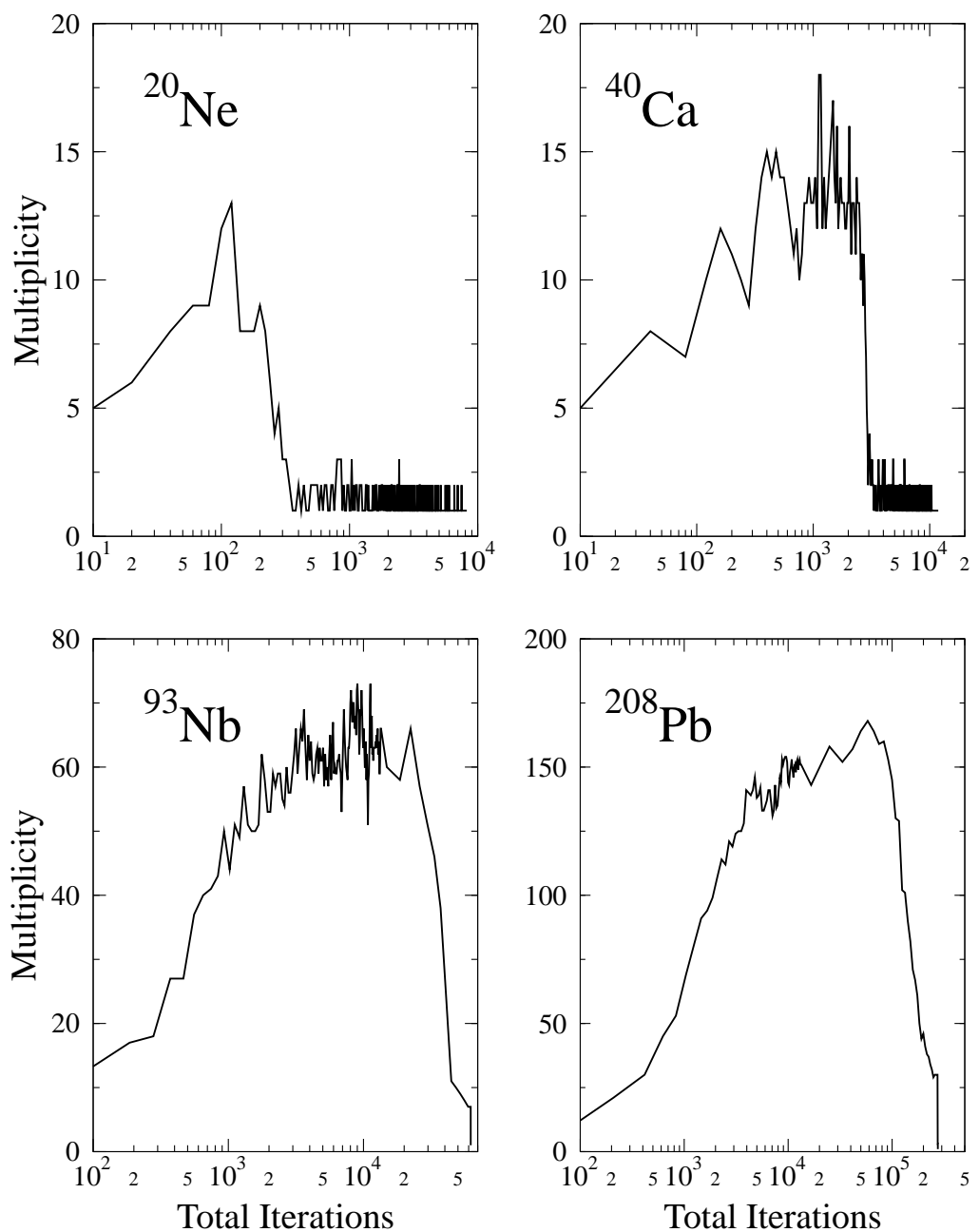

Fig. 13 

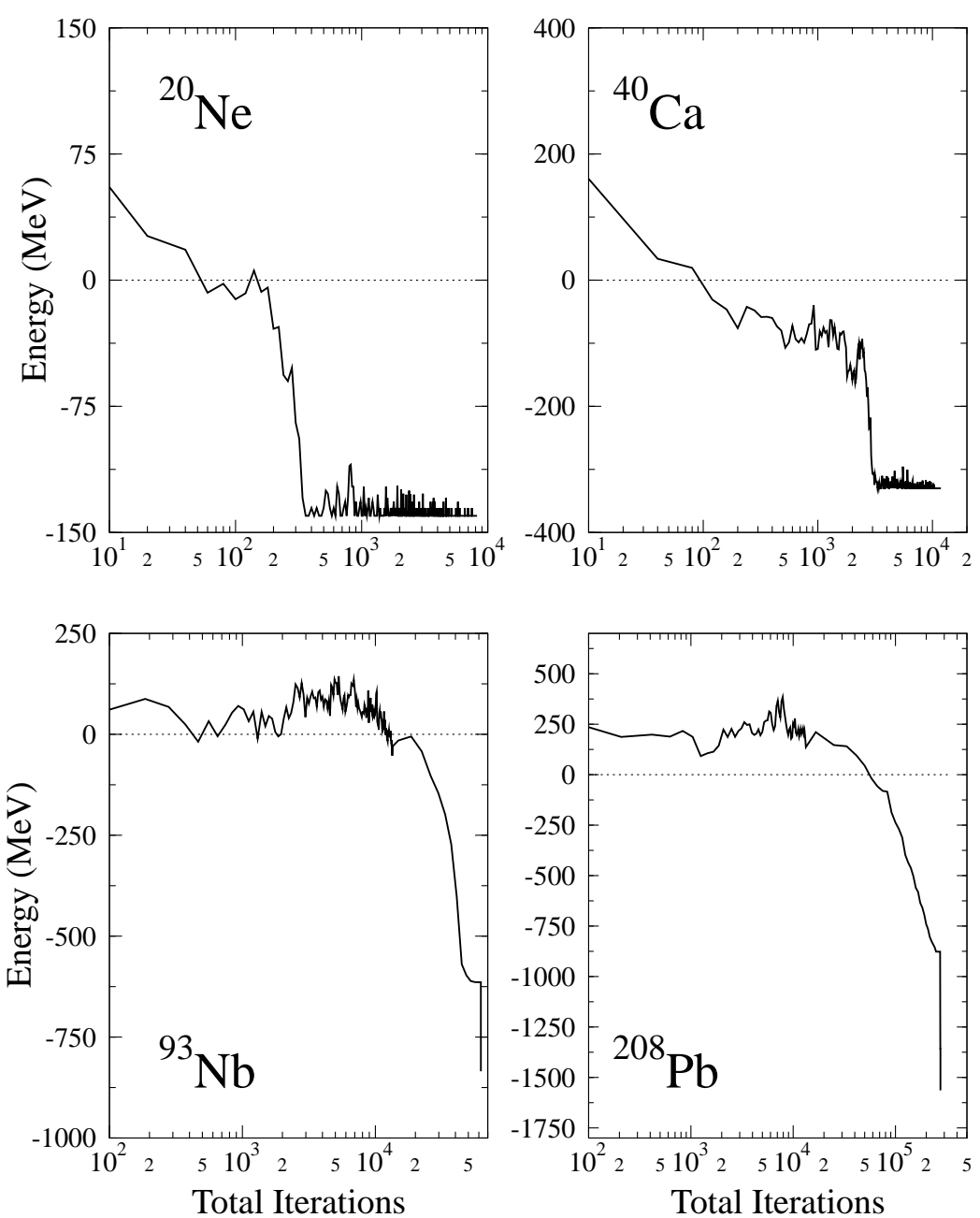

Fig. 14 


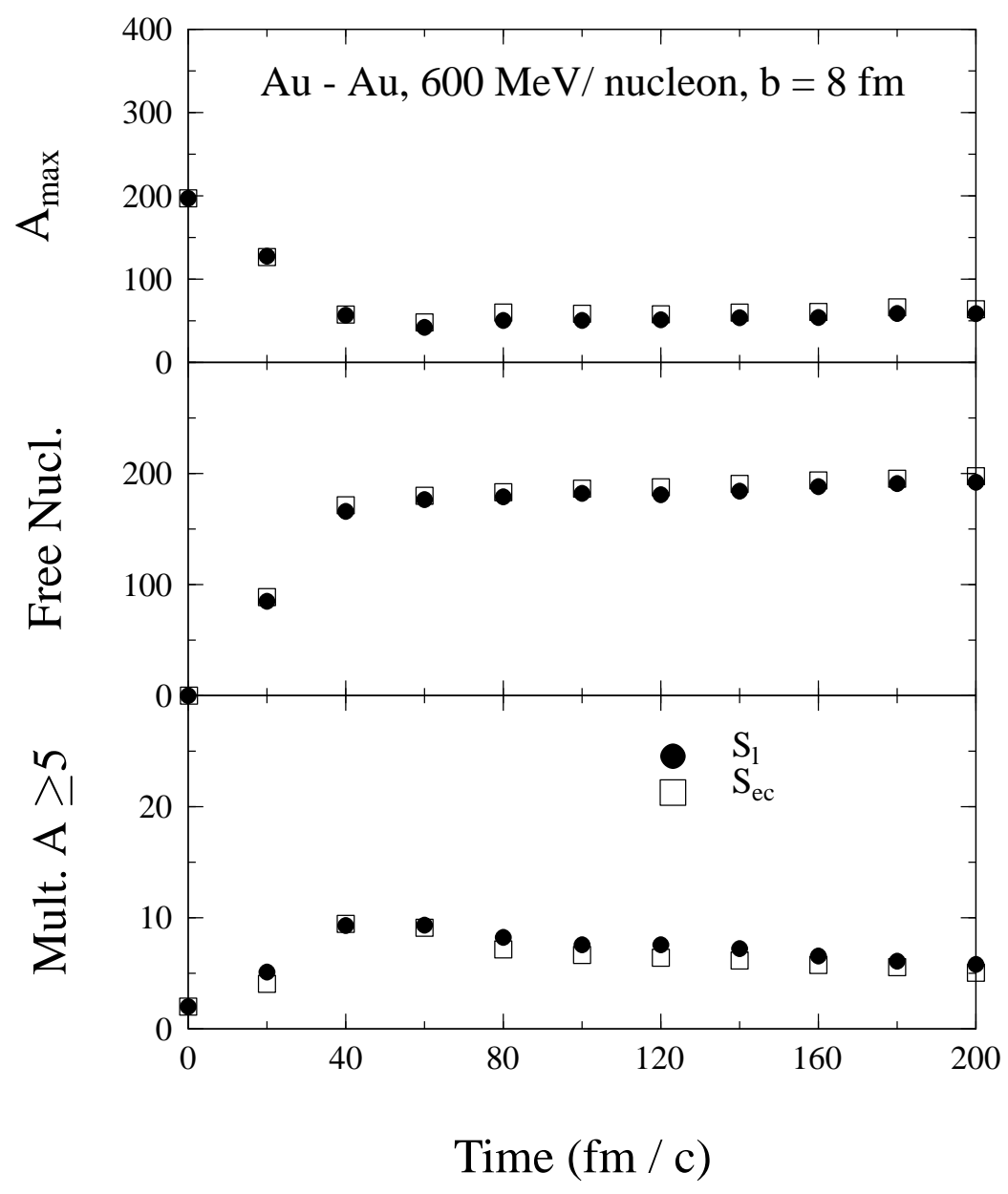

Fig. 15 